\title{
Article
}

\section{Rough Neutrosophic Digraphs with Application}

\author{
Sidra Sayed ${ }^{1}$, Nabeela Ishfaq ${ }^{1}$, Muhammad Akram ${ }^{1, *}$ (i) and Florentin Smarandache ${ }^{2}$ \\ 1 Department of Mathematics, University of the Punjab, New Campus, Lahore 54590, Pakistan; \\ sidratulmuntha228@yahoo.com (S.S.); nabeelaishfaq123@gmail.com (N.I.) \\ 2 Mathematics \& Science Department, University of New Mexico, 705 Gurley Ave., Gallup, NM 87301, USA; \\ fsmarandache@gmail.com \\ * Correspondence: m.akram@pucit.edu.pk; Tel.: +92-42-99231241
}

Received: 5 December 2017; Accepted: 15 January 2018; Published: 18 January 2018

\begin{abstract}
A rough neutrosophic set model is a hybrid model which deals with vagueness by using the lower and upper approximation spaces. In this research paper, we apply the concept of rough neutrosophic sets to graphs. We introduce rough neutrosophic digraphs and describe methods of their construction. Moreover, we present the concept of self complementary rough neutrosophic digraphs. Finally, we consider an application of rough neutrosophic digraphs in decision-making.
\end{abstract}

Keywords: rough neutrosophic sets; rough neutrosophic digraphs; decision-making

MSC: 03E72, 68R10, 68R05

\section{Introduction}

Smarandache [1] proposed the concept of neutrosophic sets as an extension of fuzzy sets [2]. A neutrosophic set has three components, namely, truth membership, indeterminacy membership and falsity membership, in which each membership value is a real standard or non-standard subset of the nonstandard unit interval $] 0-, 1+\left[([3])\right.$, where $0^{-}=0-\epsilon, 1^{+}=1+\epsilon, \epsilon$ is an infinitesimal number $>0$. To apply neutrosophic set in real-life problems more conveniently, Smarandache [3] and Wang et al. [4] defined single-valued neutrosophic sets which takes the value from the subset of $[0,1]$. Actually, the single valued neutrosophic set was introduced for the first time by Smarandache in 1998 in [3]. Ye [5] considered multicriteria decision-making method using the correlation coefficient under single-valued neutrosophic environment. Ye [6] also presented improved correlation coefficients of single valued neutrosophic sets and interval neutrosophic sets for multiple attribute decision making.

Rough set theory was proposed by Pawlak [7] in 1982. Rough set theory is useful to study the intelligence systems containing incomplete, uncertain or inexact information. The lower and upper approximation operators of rough sets are used for managing hidden information in a system. Therefore, many hybrid models have been built, such as soft rough sets, rough fuzzy sets, fuzzy rough sets, soft fuzzy rough sets, neutrosophic rough sets, andrough neutrosophic sets, for handling uncertainty and incomplete information effectively. Dubois and Prade [8] introduced the notions of rough fuzzy sets and fuzzy rough sets. Liu and Chen [9] have studied different decision-making methods. Broumi et al. [10] introduced the concept of rough neutrosophic sets. Yang et al. [11] proposed single valued neutrosophic rough sets by combining single valued neutrosophic sets and rough sets, and established an algorithm for decision-making problem based on single valued neutrosophic rough sets on two universes. Mordeson and Peng [12] presented operations on fuzzy graphs. Akram et al. [13-16] considered several new concepts of neutrosophic graphs with applications. Zafer and Akram [17] introduced a novel decision-making method based on rough fuzzy information. In this research study, we apply the concept of rough neutrosophic sets to graphs. We introduce rough neutrosophic digraphs and describe methods of their construction. Moreover, 
we present the concept of self complementary rough neutrosophic digraphs. We also present an application of rough neutrosophic digraphs in decision-making.

We have used standard definitions and terminologies in this paper. For other notations, terminologies and applications not mentioned in the paper, the readers are referred to [18-22].

\section{Rough Neutrosophic Digraphs}

Definition 1. [4] Let $Z$ be a nonempty universe. A neutrosophic set $N$ on $Z$ is defined as follows:

$$
N=\left\{<x: \mu_{N}(x), \sigma_{N}(x), \lambda_{N}(x)>, x \in Z\right\}
$$

where the functions $\mu, \sigma, \lambda: Z \rightarrow[0,1]$ represent the degree of membership, the degree of indeterminacy and the degree of falsity.

Definition 2. [7] Let $Z$ be a nonempty universe and $R$ an equivalence relation on $Z$.A pair $(Z, R)$ is called an approximation space. Let $N^{*}$ be a subset of $Z$ and the lower and upper approximations of $N^{*}$ in the approximation space $(Z, R)$ denoted by $\underline{R} N^{*}$ and $\bar{R} N^{*}$ are defined as follows:

$$
\begin{aligned}
\underline{R} N^{*} & =\left\{x \in Z \mid[x]_{R} \subseteq N^{*}\right\}, \\
\bar{R} N^{*} & =\left\{x \in Z \mid[x]_{R} \subseteq N^{*}\right\},
\end{aligned}
$$

where $[x]_{R}$ denotes the equivalence class of $R$ containing $x$. A pair $\left(\underline{R} N^{*}, \bar{R} N^{*}\right)$ is called a rough set.

Definition 3. [10] Let $Z$ be a nonempty universe and $R$ an equivalence relation on $Z$. Let $N$ be a neutrosophic set(NS) on $Z$. The lower and upper approximations of $N$ in the approximation space $(Z, R)$ denoted by $\underline{R} N$ and $\bar{R} N$ are defined as follows:

$$
\begin{aligned}
\underline{R} N & =\left\{<x, \mu_{\underline{R}(N)}(x), \sigma_{\underline{R}(N)}(x), \lambda_{\underline{R}(N)}(x)>: y \in[x]_{R}, x \in Z\right\}, \\
\bar{R} N & =\left\{<x, \mu_{\bar{R}(N)}(x), \sigma_{\bar{R}(N)}(x), \lambda_{\bar{R}(N)}(x)>: y \in[x]_{R}, x \in Z\right\},
\end{aligned}
$$

where,

$$
\begin{array}{ll}
\mu_{\underline{R}(N)}(x)=\bigwedge_{y \in[x]_{R}} \mu_{N}(y), & \mu_{\bar{R}(N)}(x)=\bigvee_{y \in[x]_{R}} \mu_{N}(y), \\
\sigma_{\underline{R}(N)}(x)=\bigwedge_{y \in[x]_{R}} \sigma_{N}(y), & \sigma_{\bar{R}(N)}(x)=\bigvee_{y \in[x]_{R}} \sigma_{N}(y, \\
\lambda_{\underline{R}(N)}(x)=\bigvee_{y \in[x]_{R}} \lambda_{N}(y), & \lambda_{\bar{R}(N)}(x)=\bigwedge_{y \in[x]_{R}} \lambda_{N}(y) .
\end{array}
$$

A pair $(\underline{R} N, \bar{R} N)$ is called a rough neutrosophic set.

We now define the concept of rough neutrosophic digraph.

Definition 4. Let $V^{*}$ be a nonempty set and $\mathrm{R}$ an equivalence relation on $V^{*}$. Let $V$ be a NS on $V^{*}$, defined as

$$
V=\left\{<x, \mu_{V}(x), \sigma_{V}(x), \lambda_{V}(x)>: x \in V^{*}\right\} .
$$

Then, the lower and upper approximations of $V$ represented by $\underline{R} V$ and $\bar{R} V$, respectively, are characterized as NSs in $V^{*}$ such that $\forall x \in V^{*}$,

$$
\begin{aligned}
\underline{R}(V) & =\left\{<x, \mu_{\underline{R}(V)}(x), \sigma_{\underline{R}(V)}(x), \lambda_{\underline{R}(V)}(x)>: y \in[x]_{R}\right\}, \\
\bar{R}(V) & =\left\{<x, \mu_{\bar{R}(V)}(x), \sigma_{\bar{R}(V)}(x), \lambda_{\bar{R}(V)}(x)>: y \in[x]_{R}\right\},
\end{aligned}
$$

where,

$$
\begin{array}{ll}
\mu_{\underline{R}(V)}(x)=\bigwedge_{y \in[x]_{R}} \mu_{V}(y), & \mu_{\bar{R}(V)}(x)=\bigvee_{y \in[x]_{R}} \mu_{V}(y), \\
\sigma_{\underline{R}(V)}(x)=\bigwedge_{y \in[x]_{R}} \sigma_{V}(y), & \sigma_{\bar{R}(V)}(x)=\bigvee_{y \in[x]_{R}} \sigma_{V}(y), \\
\lambda_{\underline{R}(V)}(x)=\bigvee_{y \in[x]_{R}} \lambda_{V}(y), & \lambda_{\bar{R}(V)}(x)=\bigwedge_{y \in[x]_{R}} \lambda_{V}(y) .
\end{array}
$$


Let $E^{*} \subseteq V^{*} \times V^{*}$ and $S$ an equivalence relation on $E^{*}$ such that

$$
\left(\left(x_{1}, x_{2}\right),\left(y_{1}, y_{2}\right)\right) \in S \Leftrightarrow\left(x_{1}, y_{1}\right),\left(x_{2}, y_{2}\right) \in R .
$$

Let $E$ be a neutrosophic set on $E^{*} \subseteq V^{*} \times V^{*}$ defined as

$$
E=\left\{<x y, \mu_{E}(x y), \sigma_{E}(x y), \lambda_{E}(x y)>: x y \in V^{*} \times V^{*}\right\},
$$

such that

$$
\begin{aligned}
& \mu_{E}(x y) \leq \min \left\{\mu_{\underline{R} V}(x), \mu_{\underline{R} V}(y)\right\} \\
& \sigma_{E}(x y) \leq \min \left\{\sigma_{\underline{R} V}(x), \sigma_{\underline{R} V}(y)\right\} \\
& \lambda_{E}(x y) \leq \max \left\{\lambda_{\bar{R} V}(x), \lambda_{\bar{R} V}(y)\right\} \quad \forall x, y \in V^{*} .
\end{aligned}
$$

Then, the lower and upper approximations of $E$ represented by $\underline{S} E$ and $\bar{S} E$, respectively, are defined as follows

$$
\begin{aligned}
& \underline{S} E=\left\{<x y, \mu_{\underline{S} E}(x y), \sigma_{\underline{S} E}(x y), \lambda_{\underline{S} E}(x y)>: w z \in[x y]_{S}, x y \in V^{*} \times V^{*}\right\}, \\
& \bar{S} E=\left\{<x y, \mu_{\bar{S} E}(x y), \sigma_{\bar{S} E}(x y), \lambda_{\bar{S} E}(x y)>: w z \in[x y]_{S}, x y \in V^{*} \times V^{*}\right\},
\end{aligned}
$$

where,

$$
\begin{array}{lll}
\mu_{\underline{S}(E)}(x y) & =\bigwedge_{w z \in[x y]_{S}} \mu_{E}(w z), & \mu_{\bar{S}(E)}(x y)=\bigvee_{w z \in[x y]_{S}} \mu_{E}(w z), \\
\sigma_{\underline{S}(E)}(x y)=\bigwedge_{w z \in[x y]_{S}} \sigma_{E}(w z), & \sigma_{\bar{S}(E)}(x y)=\bigvee_{w z \in[x y]_{S}} \sigma_{E}(w z) \\
\lambda_{\underline{S}(E)}(x y)=\bigvee_{w z \in[x y]_{S}} \lambda_{E}(w z), & \lambda_{\bar{S}(E)}(x y)=\bigwedge_{w z \in[x y]_{S}} \lambda_{E}(w z) .
\end{array}
$$

A pair $S E=(\underline{S} E, \bar{S} E)$ is called a rough neutrosophic relation.

Definition 5. A rough neutrosophic digraph on a nonempty set $V^{*}$ is a four-ordered tuple $G=(R, R V, S, S E)$ such that

(a) $R$ is an equivalence relation on $V^{*}$;

(b) $S$ is an equivalence relation on $E^{*} \subseteq V^{*} \times V^{*}$;

(c) $R V=(\underline{R} V, \bar{R} V)$ is a rough neutrosophic set on $V^{*}$;

(d) $S E=(\underline{S E}, \bar{S} E)$ is a rough neutrosophic relation on $V^{*}$ and

(e) $(R V, S E)$ is a neutrosophic digraph where $\underline{G}=(\underline{R} V, \underline{S} E)$ and $\bar{G}=(\bar{R} V, \bar{S} E)$ are lower and upper approximate neutrosophic digraphs of $G$ such that

$$
\begin{aligned}
& \mu_{\underline{S} E}(x y) \leq \min \left\{\mu_{\underline{R} V}(x), \mu_{\underline{R} V}(y)\right\}, \\
& \sigma_{\underline{S} E}(x y) \leq \min \left\{\sigma_{\underline{R} V}(x), \sigma_{\underline{R} V}(y)\right\}, \\
& \lambda_{\underline{S} E}(x y) \leq \max \left\{\lambda_{\underline{R} V}(x), \lambda_{\underline{R} V}(y)\right\},
\end{aligned}
$$

and

$$
\begin{aligned}
& \mu_{\bar{S} E}(x y) \leq \min \left\{\mu_{\bar{R} V}(x), \mu_{\bar{R} V}(y)\right\} \\
& \sigma_{\bar{S} E}(x y) \leq \min \left\{\sigma_{\bar{R} V}(x), \sigma_{\bar{R} V}(y)\right\} \\
& \lambda_{\bar{S} E}(x y) \leq \max \left\{\lambda_{\bar{R} V}(x), \lambda_{\bar{R} V}(y)\right\} \forall x, y \in V^{*}
\end{aligned}
$$

Example 1. Let $V^{*}=\{a, b, c\}$ be a set and $R$ an equivalence relation on $V^{*}$

$$
R=\left[\begin{array}{lll}
1 & 0 & 1 \\
0 & 1 & 0 \\
1 & 0 & 1
\end{array}\right]
$$


Let $V=\{(a, 0.2,0.3,0.6),(b, 0.8,0.6,0.5),(c, 0.9,0.1,0.4)\}$ be a neutrosophic set on $V^{*}$. The lower and upper approximations of $V$ are given by,

$$
\begin{aligned}
& \underline{R} V=\{(a, 0.2,0.1,0.6),(b, 0.8,0.6,0.5),(c, 0.2,0.1,0.6)\}, \\
& \bar{R} V=\{(a, 0.9,0.3,0.4),(b, 0.8,0.6,0.5),(c, 0.9,0.3,0.4)\} .
\end{aligned}
$$

Let $E^{*}=\{a a, a b, a c, b b, c a, c b\} \subseteq V^{*} \times V^{*}$ and $S$ an equivalence relation on $E^{*}$ defined as:

$$
S=\left[\begin{array}{llllll}
1 & 0 & 1 & 0 & 1 & 0 \\
0 & 1 & 0 & 0 & 0 & 1 \\
1 & 0 & 1 & 0 & 1 & 0 \\
0 & 0 & 0 & 1 & 0 & 0 \\
1 & 0 & 1 & 0 & 1 & 0 \\
0 & 1 & 0 & 0 & 0 & 1
\end{array}\right]
$$

Let $E=\{(a a, 0.2,0.1,0.4),(a b, 0.2,0.1,0.5),(a c, 0.1,0.1,0.5),(b b, 0.7,0.5,0.5),(c a, 0.1,0.1,0.3)$, $(c b, 0.2,0.1,0.5)\}$ be a neutrosophic set on $E^{*}$ and $S E=(\underline{S} E, \bar{S} E)$ a rough neutrosophic relation where $\underline{S} E$ and $\bar{S} E$ are given as

$$
\begin{aligned}
\underline{S} E= & \{(a a, 0.1,0.1,0.5),(a b, 0.2,0.1,0.5),(a c, 0.1,0.1,0.5),(b b, 0.7,0.5,0.5), \\
& (c a, 0.1,0.1,0.5),(c b, 0.2,0.1,0.5)\}, \\
\bar{S} E= & \{(a a, 0.2,0.1,0.3),(a b, 0.2,0.1,0.5),(a c, 0.2,0.1,0.3),(b b, 0.7,0.5,0.5), \\
& (c a, 0.2,0.1,0.3),(c b, 0.2,0.1,0.5)\}
\end{aligned}
$$

Thus, $\underline{G}=(\underline{R} V, \underline{S} E)$ and $\bar{G}=(\bar{R} V, \bar{S} E)$ are neutrosophic digraphs as shown in Figure 1.
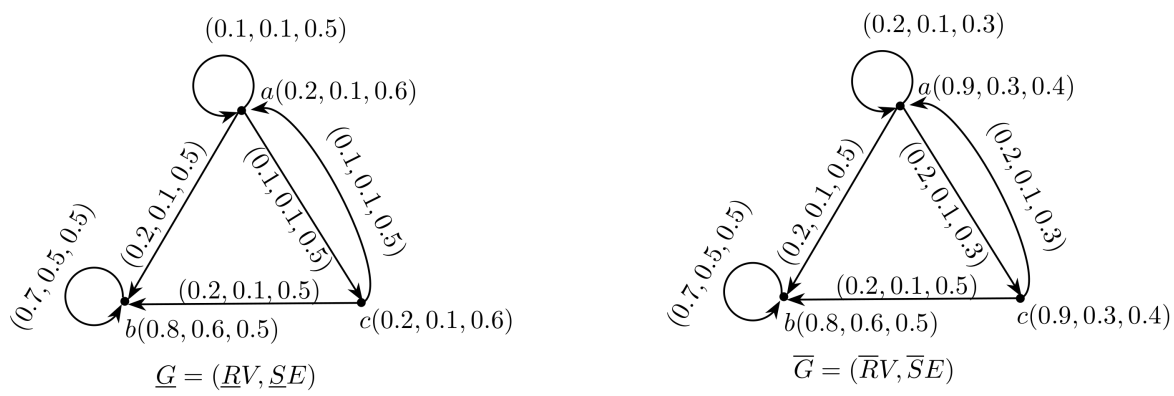

Figure 1. Rough neutrosophic digraph $G=(\underline{G}, \bar{G})$.

We now form new rough neutrosophic digraphs from old ones.

Definition 6. Let $G_{1}=\left(\underline{G}_{1}, \bar{G}_{1}\right)$ and $G_{2}=\left(\underline{G}_{2}, \bar{G}_{2}\right)$ be two rough neutrosophic digraphs on a set $V^{*}$. Then, the intersection of $G_{1}$ and $G_{2}$ is a rough neutrosophic digraph $G=G_{1} \cap G_{2}=\left(\underline{G}_{1} \cap \underline{G}_{2}, \bar{G}_{1} \cap \bar{G}_{2}\right)$, where $\underline{G}_{1} \cap \underline{G}_{2}=\left(\underline{R} V_{1} \cap \underline{R} V_{2}, \underline{S} E_{1} \cap \underline{S} E_{2}\right)$ and $\bar{G}_{1} \cap \bar{G}_{2}=\left(\bar{R} V_{1} \cap \bar{R} V_{2}, \bar{S} E_{1} \cap \bar{S} E_{2}\right)$ are neutrosophic digraphs, respectively, such that 
(1) $\mu_{\underline{R} V_{1} \cap \underline{R} V_{2}}(x)=\min \left\{\mu_{\underline{R} V_{1}}(x), \mu_{\underline{R} V_{2}}(x)\right\}$,

$\sigma_{\underline{R} V_{1} \cap \underline{R} V_{2}}(x)=\min \left\{\sigma_{\underline{R} V_{1}}(x), \sigma_{\underline{R} V_{2}}(x)\right\}$,

$\lambda_{\underline{R} V_{1} \cap \underline{R} V_{2}}(x)=\max \left\{\lambda_{\underline{R} V_{1}}(x), \lambda_{\underline{R} V_{2}}(x)\right\} \quad \forall x \in \underline{R} V_{1} \cap \underline{R} V_{1}$,

$\mu_{\underline{S} E_{1} \cap \underline{S} E_{2}}(x y)=\min \left\{\mu_{\underline{S} E_{1}}(x), \mu_{\underline{S} E_{2}}(y)\right\}$,

$\sigma_{\underline{S} E_{1} \cap \underline{S} E_{2}}(x y)=\min \left\{\sigma_{\underline{S} E_{1}}(x), \sigma_{\underline{S} E_{2}}(y)\right\}$

$\lambda_{\underline{S} E_{1} \cap \underline{S}_{2}}(x y)=\max \left\{\lambda_{\underline{S} E_{1}}(x), \lambda_{\underline{S} E_{2}}(y)\right\} \quad \forall x y \in \underline{S} E_{1} \cap \underline{S} E_{2}$,

(2) $\mu_{\bar{R} V_{1} \cap \bar{R} V_{2}}(x)=\min \left\{\mu_{\bar{R} V_{1}}(x), \mu_{\bar{R} V_{2}}(x)\right\}$,

$\sigma_{\bar{R} V_{1} \cap \bar{R} V_{2}}(x)=\min \left\{\sigma_{\bar{R} V_{1}}(x), \sigma_{\bar{R} V_{2}}(x)\right\}$,

$\lambda_{\bar{R} V_{1} \cap \bar{R} V_{2}}(x)=\max \left\{\lambda_{\bar{R} V_{1}}(x), \lambda_{\bar{R} V_{2}}(x)\right\} \quad \forall x \in \bar{R} V_{1} \cap \bar{R} V_{2}$ ，

$\mu_{\bar{S}_{E_{1}} \cap \bar{S}_{E_{2}}}(x y)=\min \left\{\mu_{\bar{S}_{E_{1}}}(x), \mu_{\bar{S}_{E_{2}}}(y)\right\}$

$\sigma_{\bar{S} E_{1} \cap \bar{S} E_{2}}(x y)=\min \left\{\sigma_{\bar{S} E_{1}}(x), \sigma_{\bar{S} E_{2}}(y)\right\}$

$\lambda_{\bar{S} E_{1} \cap \bar{S} E_{2}}(x y)=\max \left\{\lambda_{\bar{S} E_{1}}(x), \lambda_{\bar{S} E_{2}}(y)\right\} \quad \forall x y \in \bar{S} E_{1} \cap \bar{S} E_{2}$.

Example 2. Consider the two rough neutrosophic digraphs $\underline{G}_{1}$ and $\bar{G}_{2}$ as shown in Figures 1 and 2. The intersection of $\underline{G}_{1}$ and $\bar{G}_{2}$ is $G=G_{1} \cap G_{2}=\left(\underline{G}_{1} \cap \underline{G}_{2}, \bar{G}_{1} \cap \bar{G}_{2}\right)$ where $\underline{G}_{1} \cap \underline{G}_{2}=\left(\underline{R} V_{1} \cap \underline{R} V_{2}, \underline{S} E_{1} \cap \underline{S} E_{2}\right)$ and $\bar{G}_{1} \cap \bar{G}_{2}=\left(\bar{R} V_{1} \cap \bar{R} V_{2}, \bar{S} E_{1} \cap \bar{S} E_{2}\right)$ are neutrosophic digraphs as shown in Figure 3.
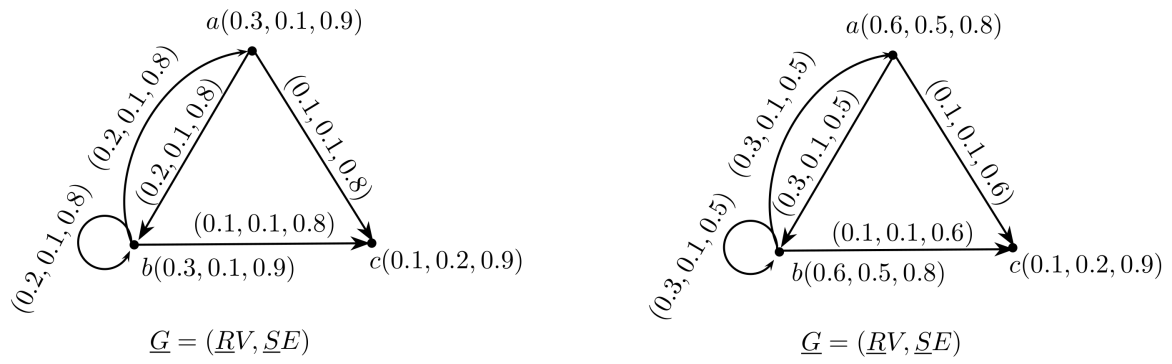

Figure 2. Rough neutrosophic digraph $G=(\underline{G}, \bar{G})$.
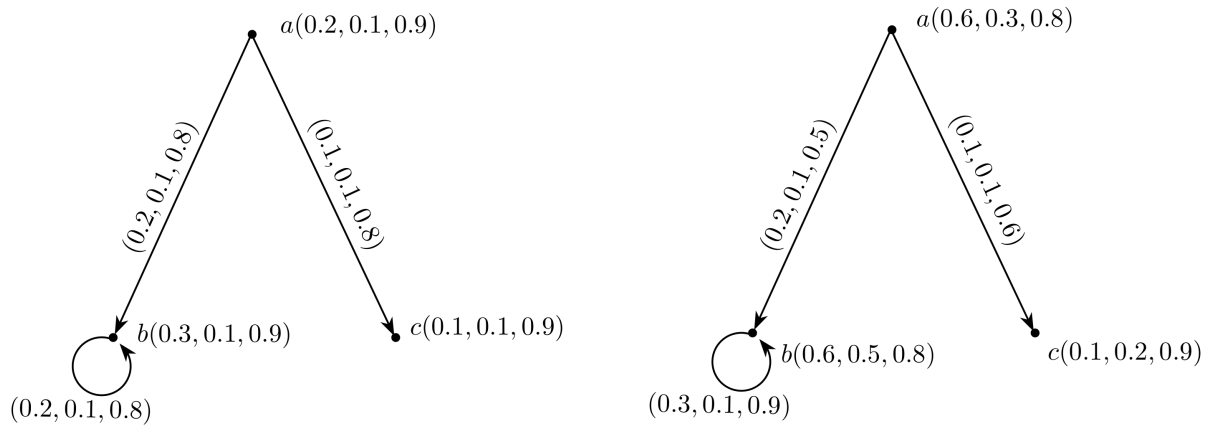

Figure 3. Rough neutrosophic digraph $G_{1} \cap G_{2}=\left(\underline{G}_{1} \cap \underline{G}_{2}, \bar{G}_{1} \cap \bar{G}_{2}\right)$.

Theorem 1. The intersection of two rough neutrosophic digraphs is a rough neutrosophic digraph.

Proof. Let $G_{1}=\left(\underline{G}_{1}, \bar{G}_{1}\right)$ and $G_{2}=\left(\underline{G}_{2}, \bar{G}_{2}\right)$ be two rough neutrosophic digraphs. Let $G=G_{1} \cap G_{2}=$ $\left(\underline{G}_{1} \cap \underline{G}_{2}, \bar{G}_{1} \cap \bar{G}_{2}\right)$ be the intersection of $G_{1}$ and $G_{2}$, where $\underline{G}_{1} \cap \underline{G}_{2}=\left(\underline{R} V_{1} \cap \underline{R} V_{2}, \underline{S} E_{1} \cap, \underline{S} E_{2}\right)$ and $\bar{G}_{1} \cap \bar{G}_{2}=\left(\bar{R} V_{1} \cap \bar{R} V_{2}, \bar{S} E_{1} \cap \bar{S} E_{2}\right)$. To prove that $G=\underline{G}_{1} \cap \bar{G}_{2}$ is a rough neutrosophic digraph, it is 
enough to show that $\underline{S} E_{1} \cap \underline{S} E_{2}$ a nd $\bar{S} E_{1} \cap \bar{S} E_{2}$ are neutrosophic relation on $\underline{R} V_{1} \cap \underline{R} V_{2}$ and $\bar{R} V_{1} \cap \bar{R} V_{2}$, respectively. First, we show that $\underline{S} E_{1} \cap \underline{S} E_{2}$ is a neutrosophic relation on $\underline{R} V_{1} \cap \underline{R} V_{2}$.

$$
\begin{aligned}
& \mu_{\underline{S} E_{1} \cap \underline{S} E_{2}}(x y)=\mu_{\underline{S} E_{1}}(x y) \wedge \mu_{\underline{S} E_{2}}(x y) \\
& \leq\left(\mu_{\underline{R} V_{1}}(x) \wedge \mu_{\underline{R} V_{2}}(y)\right) \wedge\left(\mu_{\underline{R} V_{1}}(x) \wedge \mu_{\underline{R} V_{2}}(y)\right) \\
& =\left(\mu_{\underline{R} V_{1}}(x) \wedge \mu_{\underline{R} V_{2}}(x)\right) \wedge\left(\mu_{\underline{R} V_{1}}(y) \wedge \mu_{\underline{R} V_{2}}(y)\right. \\
& =\mu_{\underline{R} V_{1} \cap \underline{R} V_{2}}(x) \wedge \mu_{\underline{R} V_{1} \cap \underline{R} V_{2}}(y) \\
& \mu_{\underline{S} E_{1} \cap \underline{S} E_{2}}(x y) \leq \min \left\{\mu_{\underline{R} V_{1} \cap \underline{R} V_{2}}(x), \mu_{\underline{R} V_{1} \cap \underline{R} V_{2}}(y)\right\} \\
& \sigma_{\underline{S} E_{1} \cap \underline{S} E_{2}}(x y)=\sigma_{\underline{S} E_{1}}(x y) \wedge \sigma_{\underline{S} E_{2}}(x y) \\
& \leq\left(\sigma_{\underline{R} V_{1}}(x) \wedge \sigma_{\underline{R} V_{2}}(y)\right) \wedge\left(\sigma_{\underline{R} V_{1}}(x) \wedge \sigma_{\underline{R} V_{2}}(y)\right) \\
& =\left(\sigma_{\underline{R} V_{1}}(x) \wedge \sigma_{\underline{R} V_{2}}(x)\right) \wedge\left(\sigma_{\underline{R} V_{1}}(y) \wedge \sigma_{\underline{R} V_{2}}(y)\right. \\
& =\sigma_{\underline{R} V_{1} \cap \underline{R} V_{2}}(x) \wedge \sigma_{\underline{R} V_{1} \cap \underline{R} V_{2}}(y) \\
& \sigma_{\underline{S} E_{1} \cap \underline{S} E_{2}}(x y) \leq \min \left\{\sigma_{\underline{R} V_{1} \cap \underline{R} V_{2}}(x), \sigma_{\underline{R} V_{1} \cap \underline{R} V_{2}}(y)\right\} \\
& \lambda_{\underline{S} E_{1} \cap \underline{S} E_{2}}(x y)=\lambda_{\underline{S} E_{1}}(x y) \wedge \lambda_{\underline{S} E_{2}}(x y) \\
& \leq\left(\lambda_{\underline{R} V_{1}}(x) \vee \lambda_{\underline{R} V_{2}}(y)\right) \wedge\left(\lambda_{\underline{R} V_{1}}(x) \vee \lambda_{\underline{R} V_{2}}(y)\right) \\
& =\left(\lambda_{\underline{R} V_{1}}(x) \wedge \lambda_{\underline{R} V_{2}}(x)\right) \vee\left(\lambda_{\underline{R} V_{1}}(y) \wedge \lambda_{\underline{R} V_{2}}(y)\right. \\
& =\lambda_{\underline{R} V_{1} \cap \underline{R} V_{2}}(x) \vee \lambda_{\underline{R} V_{1} \cap \underline{R} V_{2}}(y) \\
& \lambda_{\underline{S} E_{1} \cap \underline{S} E_{2}}(x y) \leq \max \left\{\lambda_{\underline{R} V_{1} \cap \underline{R} V_{2}}(x), \lambda_{\underline{R} V_{1} \cap \underline{R} V_{2}}(y)\right\} \text {. }
\end{aligned}
$$

Thus, from above it is clear that $\underline{S} E_{1} \cap \underline{S} E_{2}$ is a neutrosophic relation on $\underline{R} V_{1} \cap \underline{R} V_{2}$.

Similarly, we can show that $\bar{S} E_{1} \cap \bar{S} E_{2}$ is a neutrosophic relation on $\bar{R} V_{1} \cap \bar{R} V_{2}$. Hence, $G$ is a rough neutrosophic digraph.

Definition 7. The Cartesian product of two neutrosophic digraphs $G_{1}$ and $G_{2}$ is a rough neutrosophic digraph $G=G_{1} \ltimes G_{2}=\left(\underline{G}_{1} \ltimes \underline{G}_{2}, \bar{G}_{1} \ltimes \bar{G}_{2}\right)$, where $\underline{G}_{1} \ltimes \underline{G}_{2}=\left(\underline{R}_{1} \ltimes \underline{R}_{2}, \underline{S} E_{1} \ltimes \underline{S} E_{2}\right.$ and $\bar{G}_{1} \ltimes \bar{G}_{2}=\left(\bar{R} V_{1} \ltimes\right.$ $\left.\bar{R} V_{2}, \bar{S} E_{1} \ltimes \bar{S} E_{2}\right)$ such that

$$
\text { (1) } \begin{aligned}
\mu_{\underline{R} V_{1} \ltimes \underline{R} V_{2}}\left(x_{1}, x_{2}\right) & =\min \left\{\mu_{\underline{R} V_{1}}\left(x_{1}\right), \mu_{\underline{R} V_{2}}\left(x_{2}\right)\right\}, \\
\sigma_{\underline{R} V_{1} \ltimes \underline{R} V_{2}}\left(x_{1}, x_{2}\right) & =\min \left\{\sigma_{\underline{R} V_{1}}\left(x_{1}\right), \mu_{\underline{R} V_{2}}\left(x_{2}\right)\right\}, \\
\lambda_{\underline{R} V_{1} \ltimes \underline{R} V_{2}}\left(x_{1}, x_{2}\right) & =\max \left\{\lambda_{\underline{R} V_{1}}\left(x_{1}\right), \mu_{\underline{R} V_{2}}\left(x_{2}\right)\right\}, \quad \forall\left(x_{1}, x_{2}\right) \in \underline{R} V_{1} \ltimes \underline{R} V_{2}, \\
\mu_{\underline{S} E_{1} \ltimes \underline{S} E_{2}}\left(x, x_{2}\right)\left(x, y_{2}\right) & =\min \left\{\mu_{\underline{R} V_{1}}(x), \mu_{\underline{S} E_{2}}\left(x_{2}, y_{2}\right)\right\}, \\
\sigma_{\underline{S} E_{1} \ltimes \underline{S} E_{2}}\left(x, x_{2}\right)\left(x, y_{2}\right) & =\min \left\{\sigma_{\underline{R} V_{1}}(x), \sigma_{\underline{S} E_{2}}\left(x_{2}, y_{2}\right)\right\}, \\
\lambda_{\underline{S} E_{1} \ltimes \underline{S} E_{2}}\left(x, x_{2}\right)\left(x, y_{2}\right) & =\max \left\{\lambda_{\underline{R} V_{1}}(x), \lambda_{\underline{S} E_{2}}\left(x_{2}, y_{2}\right)\right\} \quad \forall x \in \underline{R} V_{1}, x_{2} y_{2} \in \underline{S} E_{2}, \\
\mu_{\underline{S} E_{1} \ltimes \underline{S} E_{2}}\left(x_{1}, z\right)\left(y_{1}, z\right) & =\min \left\{\mu_{\underline{S} E_{1}}\left(x_{1}, y_{1}\right), \mu_{\underline{R} V_{2}}(z)\right\}, \\
\sigma_{\underline{S} E_{1} \ltimes \underline{S} E_{2}}\left(x_{1}, z\right)\left(y_{1}, z\right) & =\min \left\{\sigma_{\underline{S} E_{1}}\left(x_{1}, y_{1}\right), \sigma_{\underline{R} V_{2}}(z)\right\}, \\
\lambda_{\underline{S} E_{1} \ltimes \underline{S} E_{2}}\left(x_{1}, z\right)\left(y_{1}, z\right) & =\max \left\{\lambda_{\underline{S} E_{1}}\left(x_{1}, y_{1}\right), \lambda_{\underline{R} V_{2}}(z)\right\} \quad \forall x_{1} y_{1} \in \underline{S} E_{1}, z \in \underline{R} V_{2},
\end{aligned}
$$

$$
\begin{aligned}
\mu_{\bar{R} V_{1} \ltimes \bar{R} V_{2}}\left(x_{1}, x_{2}\right) & =\min \left\{\mu_{\bar{R} V_{1}}\left(x_{1}\right), \mu_{\bar{R} V_{2}}\left(x_{2}\right)\right\}, \\
\sigma_{\bar{R} V_{1} \ltimes \bar{R} V_{2}}\left(x_{1}, x_{2}\right) & =\min \left\{\sigma_{\bar{R} V_{1}}\left(x_{1}\right), \mu_{\bar{R} V_{2}}\left(x_{2}\right)\right\}, \\
\lambda_{\bar{R} V_{1} \ltimes \bar{R} V_{2}}\left(x_{1}, x_{2}\right) & =\max \left\{\lambda_{\bar{R} V_{1}}\left(x_{1}\right), \mu_{\bar{R} V_{2}}\left(x_{2}\right)\right\} \quad \forall\left(x_{1}, x_{2}\right) \in \bar{R} V_{1} \ltimes \bar{R} V_{2}, \\
\mu_{\bar{S} E_{1} \ltimes \bar{S} E_{2}}\left(x, x_{2}\right)\left(x, y_{2}\right) & =\min \left\{\mu_{\bar{R} V_{1}}(x), \mu_{\bar{S} E_{2}}\left(x_{2}, y_{2}\right)\right\}, \\
\sigma_{\bar{S} E_{1} \ltimes \bar{S} E_{2}}\left(x, x_{2}\right)\left(x, y_{2}\right) & =\min \left\{\sigma_{\bar{R} V_{1}}(x), \sigma_{\bar{S} E_{2}}\left(x_{2}, y_{2}\right)\right\}, \\
\lambda_{\bar{S} E_{1} \ltimes \bar{S} E_{2}}\left(x, x_{2}\right)\left(x, y_{2}\right) & =\max \left\{\lambda_{\bar{R} V_{1}}(x), \lambda_{\bar{S} E_{2}}\left(x_{2}, y_{2}\right)\right\} \quad \forall x \in \bar{R} V_{1}, x_{2} y_{2} \in \bar{S} E_{2},
\end{aligned}
$$




$$
\begin{aligned}
& \mu_{\bar{S} E_{1} \ltimes \bar{S} E_{2}}\left(x_{1}, z\right)\left(y_{1}, z\right)=\min \left\{\mu_{\bar{S} E_{1}}\left(x_{1}, y_{1}\right), \mu_{\bar{R} V_{2}}(z)\right\}, \\
& \sigma_{\bar{S} E_{1} \ltimes \bar{S} E_{2}}\left(x_{1}, z\right)\left(y_{1}, z\right)=\min \left\{\sigma_{\bar{S} E_{1}}\left(x_{1}, y_{1}\right), \sigma_{\bar{R} V_{2}}(z)\right\}, \\
& \lambda_{\bar{S} E_{1} \ltimes \bar{S} E_{2}}\left(x_{1}, z\right)\left(y_{1}, z\right)=\max \left\{\lambda_{\bar{S} E_{1}}\left(x_{1}, y_{1}\right), \lambda_{\bar{R} V_{2}}(z)\right\} \quad \forall x_{1} y_{1} \in \bar{S} E_{1}, z \in \bar{R} V_{2},
\end{aligned}
$$

Example 3. Let $V^{*}=\{a, b, c, d\}$ be a set. Let $G_{1}=\left(\underline{G}_{1}, \bar{G}_{1}\right)$ and $G_{2}=\left(\underline{G}_{2}, \bar{G}_{2}\right)$ be two rough neutrosophic digraphs on $V^{*}$, as shown in Figures 4 and 5 . The cartesian product of $G_{1}$ and $G_{2}$ is $G=\left(\underline{G}_{1} \times \underline{G}_{2}, \bar{G}_{1} \times \bar{G}_{2}\right)$, where $\underline{G}_{1} \times \underline{G}_{2}=\left(\underline{R} N_{1} \times \underline{R} N_{2}, \underline{S} E_{1} \times \underline{S} E_{2}\right)$ and $\bar{G}_{1} \times \bar{G}_{2}=\left(\bar{R} N_{1} \times \bar{R} N_{2}, \bar{S} E_{1} \times \bar{S} E_{2}\right)$ are neutrosophic digraphs, as shown in Figures 6 and 7 , respectively.
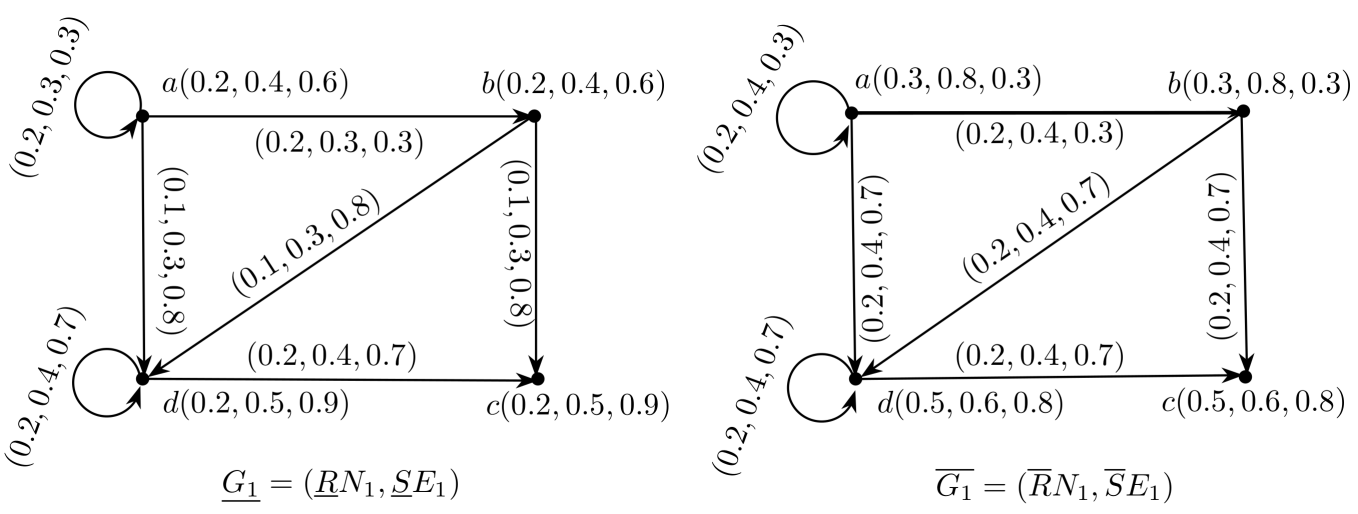

Figure 4. Rough neutrosophic digraph $G_{1}=\left(\underline{G_{1}}, \overline{G_{1}}\right)$.
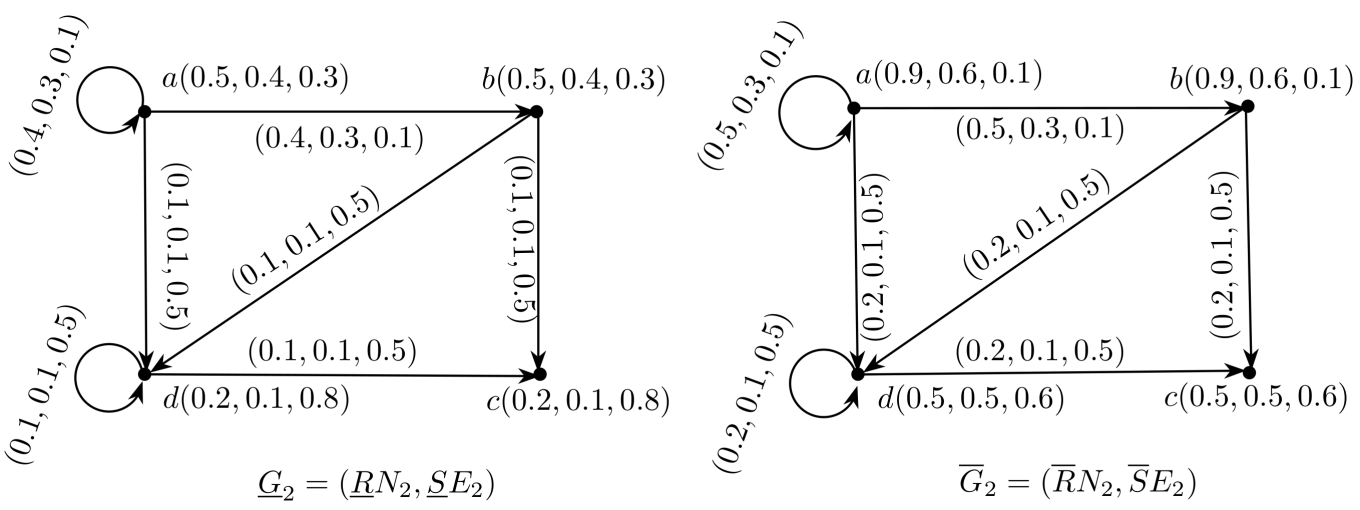

Figure 5. Rough neutrosophic digraph $G_{2}=\left(\underline{G}_{2}, \bar{G}_{2}\right)$. 


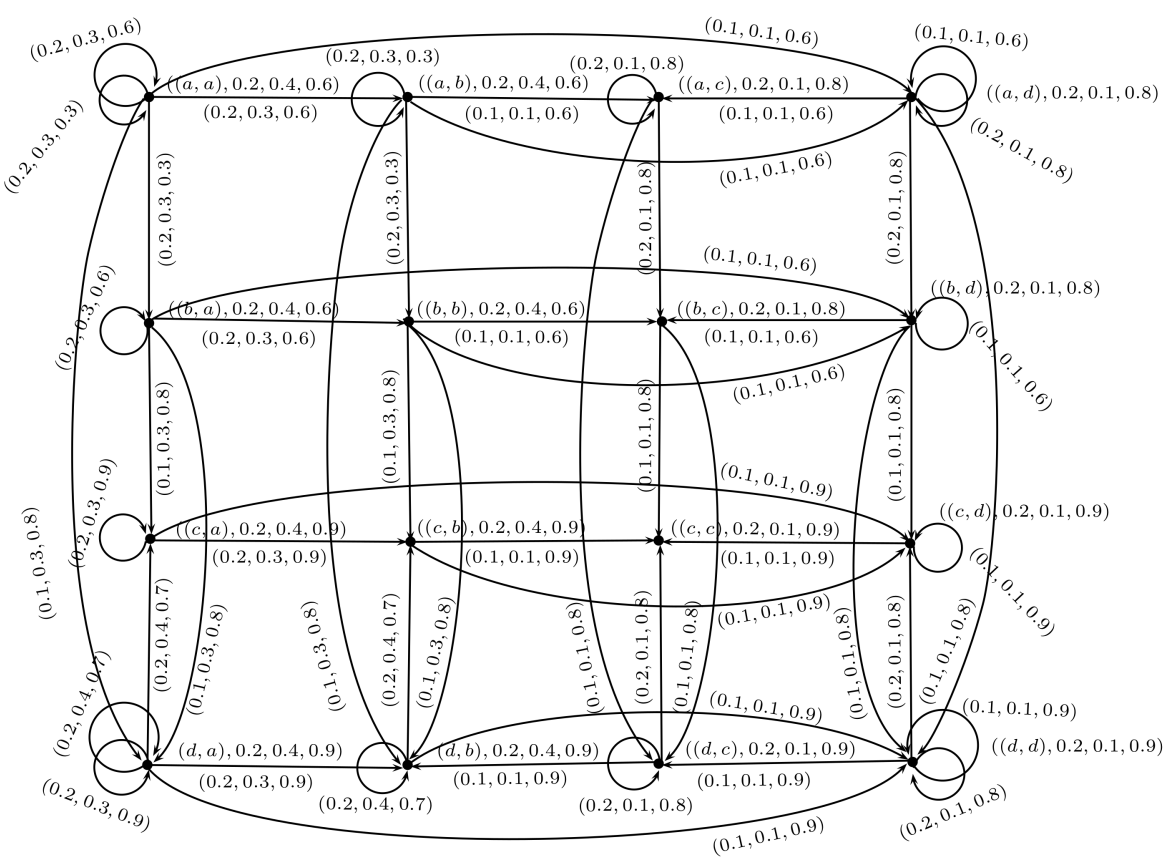

Figure 6. Neutrosophic digraph $\underline{G}_{1} \times \underline{G}_{2}=\left(\underline{R} N_{1} \times \underline{R} N_{2}, \underline{S} E_{1} \times \underline{S} E_{2}\right)$.

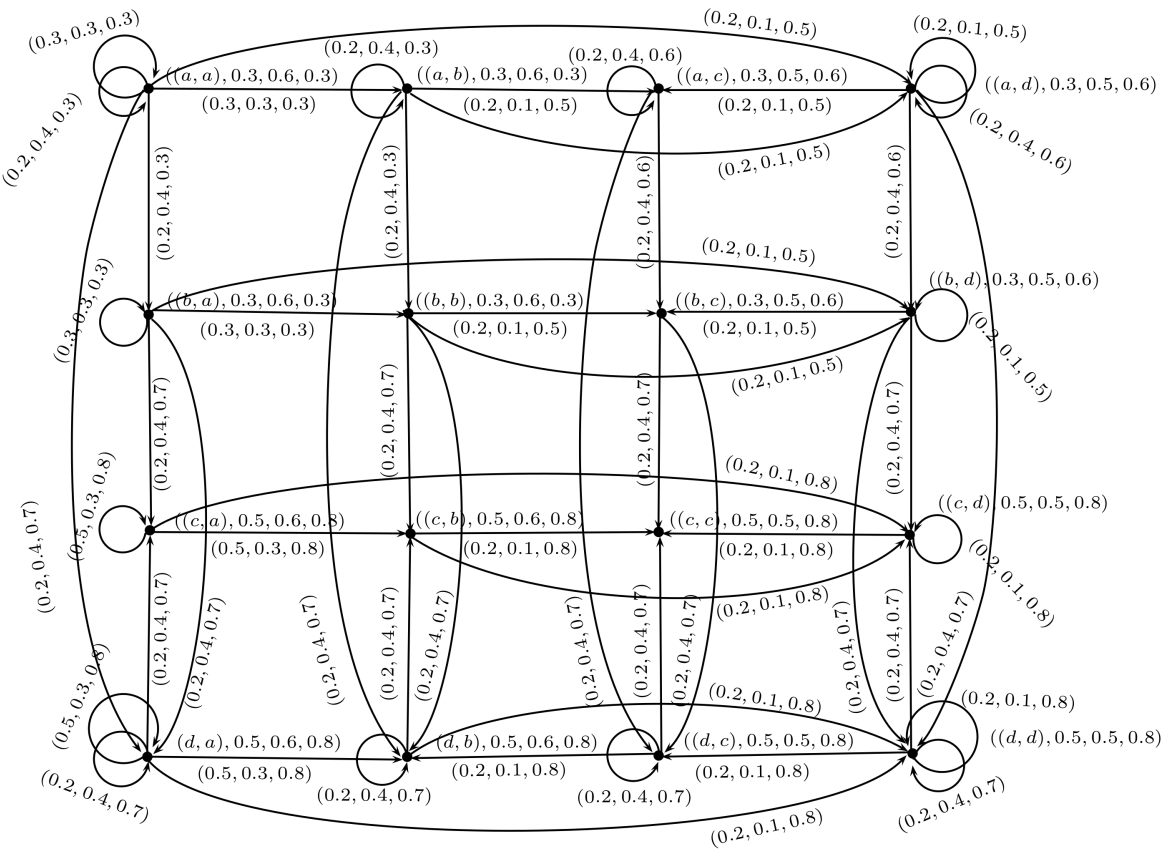

Figure 7. Neutrosophic digraph $\bar{G}_{1} \times \bar{G}_{2}=\left(\bar{R} N_{1} \times \bar{R} N_{2}, \bar{S} E_{1} \times \bar{S} E_{2}\right)$.

Theorem 2. The Cartesian product of two rough neutrosophic digraphs is a rough neutrosophic digraph.

Proof. Let $G_{1}=\left(\underline{G}_{1}, \bar{G}_{1}\right)$ and $G_{2}=\left(\underline{G}_{2}, \bar{G}_{2}\right)$ be two rough neutrosophic digraphs. Let $G=G_{1} \ltimes G_{2}=$ $\left(\underline{G}_{1} \ltimes \underline{G}_{2}, \bar{G}_{1} \ltimes \bar{G}_{2}\right)$ be the Cartesian product of $G_{1}$ and $G_{2}$, where $\underline{G}_{1} \ltimes \underline{G}_{2}=\left(\underline{R} V_{1} \ltimes \underline{R} V_{2}, \underline{S} E_{1} \ltimes \underline{S} E_{2}\right)$ and $\bar{G}_{1} \ltimes \bar{G}_{2}=\left(\bar{R} V_{1} \ltimes \bar{R} V_{2}, \bar{S} E_{1} \ltimes \bar{S} E_{2}\right)$. To prove that $G=\underline{G}_{1} \ltimes \bar{G}_{2}$ is a rough neutrosophic digraph, it is enough to show that $\underline{S} E_{1} \ltimes \underline{S} E_{2}$ and $\bar{S} E_{1} \ltimes \bar{S} E_{2}$ are neutrosophic relation on $\underline{R} V_{1} \ltimes \underline{R} V_{2}$ and $\bar{R} V_{1} \ltimes \bar{R} V_{2}$, respectively. First, we show that $\underline{S} E_{1} \ltimes \underline{S} E_{2}$ is a neutrosophic relation on $\underline{R} V_{1} \ltimes \underline{R} V_{2}$. 
If $x \in \underline{R} V_{1}, x_{2} y_{2} \in \underline{S} E_{2}$, then

$$
\begin{aligned}
& \mu_{\underline{S} E_{1} \ltimes \underline{S} E_{2}}\left(x, x_{2}\right)\left(x, y_{2}\right)=\mu_{\underline{R} V_{1}}(x) \wedge \mu_{\underline{S} E_{2}}\left(x_{2}, y_{2}\right) \\
& \leq \mu_{\underline{R} V_{1}}(x) \wedge\left(\mu_{\underline{R} V_{2}}\left(x_{2}\right) \wedge \mu_{\underline{R} V_{2}}\left(y_{2}\right)\right) \\
& =\left(\mu_{\underline{R} V_{1}}(x) \wedge \mu_{\underline{R} V_{2}}\left(x_{2}\right)\right) \wedge\left(\mu_{\underline{R} V_{1}}(x) \wedge \mu_{\underline{R} V_{2}}\left(y_{2}\right)\right) \\
& =\mu_{\underline{R} V_{1} \ltimes \underline{R} V_{2}}\left(x, x_{2}\right) \wedge \mu_{\underline{R} V_{1} \ltimes \underline{R} V_{2}}\left(x, y_{2}\right) \\
& \mu_{\underline{S} E_{1} \ltimes \underline{S} E_{2}}\left(x, x_{2}\right)\left(x, y_{2}\right) \leq \min \left\{\mu_{\underline{R} V_{1} \ltimes \underline{R} V_{2}}\left(x, x_{2}\right), \mu_{\underline{R} V_{1} \ltimes \underline{R} V_{2}}\left(x, y_{2}\right)\right\} \text {, } \\
& \sigma_{\underline{S} E_{1} \ltimes \underline{S} E_{2}}\left(x, x_{2}\right)\left(x, y_{2}\right)=\sigma_{\underline{R} V_{1}}(x) \wedge \sigma_{\underline{S}} E_{2}\left(x_{2}, y_{2}\right) \\
& \leq \sigma_{\underline{R} V_{1}}(x) \wedge\left(\sigma_{\underline{R} V_{2}}\left(x_{2}\right) \wedge \sigma_{\underline{R} V_{2}}\left(y_{2}\right)\right) \\
& =\left(\sigma_{\underline{R} V_{1}}(x) \wedge \sigma_{\underline{R} V_{2}}\left(x_{2}\right)\right) \wedge\left(\sigma_{\underline{R} V_{1}}(x) \wedge \sigma_{\underline{R} V_{2}}\left(y_{2}\right)\right. \\
& =\sigma_{\underline{R} V_{1} \ltimes \underline{R} V_{2}}\left(x, x_{2}\right) \wedge \sigma_{\underline{R} V_{1} \ltimes \underline{R} V_{2}}\left(x, y_{2}\right) \\
& \sigma_{\underline{S} E_{1} \ltimes \underline{S} E_{2}}\left(x, x_{2}\right)\left(x, y_{2}\right) \leq \min \left\{\sigma_{\underline{R} V_{1} \ltimes \underline{R} V_{2}}\left(x, x_{2}\right), \sigma_{\underline{R} V_{1} \ltimes \underline{R} V_{2}}\left(x, y_{2}\right)\right\} \text {, } \\
& \lambda_{\underline{S} E_{1} \ltimes \underline{S} E_{2}}\left(x, x_{2}\right)\left(x, y_{2}\right)=\lambda_{\underline{R} V_{1}}(x) \vee \lambda_{\underline{S} E_{2}}\left(x_{2}, y_{2}\right) \\
& \leq \lambda_{\underline{R} V_{1}}(x) \vee\left(\lambda_{\underline{R} V_{2}}\left(x_{2}\right) \vee \lambda_{\underline{R} V_{2}}\left(y_{2}\right)\right) \\
& =\left(\lambda_{\underline{R} V_{1}}(x) \vee \lambda_{\underline{R} V_{2}}\left(x_{2}\right)\right) \vee\left(\lambda_{\underline{R} V_{1}}(x) \vee \lambda_{\underline{R} V_{2}}\left(y_{2}\right)\right) \\
& =\lambda_{\underline{R} V_{1} \ltimes \underline{R} V_{2}}\left(x, x_{2}\right) \vee \lambda_{\underline{R} V_{1} \ltimes \underline{R} V_{2}}\left(x, y_{2}\right) \\
& \lambda_{\underline{S} E_{1} \ltimes \underline{S}_{2}}\left(x, x_{2}, x, y_{2}\right) \leq \max \left\{\lambda_{\underline{R} V_{1} \ltimes \underline{R} V_{2}}\left(x, x_{2}\right), \lambda_{\underline{R} V_{1} \ltimes \underline{R} V_{2}}\left(x, y_{2}\right)\right\} \text {. }
\end{aligned}
$$

If $x_{1} y_{1} \in \underline{S} E_{1}, z \in \underline{R} V_{2}$, then

$$
\begin{aligned}
& \mu_{\underline{S} E_{1} \ltimes \underline{S} E_{2}}\left(x_{1}, z\right)\left(y_{1}, z\right)=\mu_{\underline{S} E_{1}}\left(x_{1}, y_{1}\right) \wedge \mu_{\underline{R} V_{2}}(z) \\
& \leq\left(\mu_{\underline{R} V_{1}}\left(x_{1}\right) \wedge \mu_{\underline{R} V_{1}}\left(y_{1}\right)\right) \wedge \mu_{\underline{R} V_{2}}(z) \\
& =\left(\mu_{\underline{R} V_{1}}\left(x_{1}\right) \wedge \mu_{\underline{R} V_{2}}(z)\right) \wedge\left(\mu_{\underline{R} V_{1}}\left(y_{1}\right) \wedge \mu_{\underline{R} V_{2}}(z)\right) \\
& =\mu_{\underline{R} V_{1} \ltimes \underline{R} V_{2}}\left(x_{1}, z\right) \wedge \mu_{\underline{R} V_{1} \ltimes \underline{R} V_{2}}\left(y_{1}, z\right) \\
& \mu_{\underline{S} E_{1} \ltimes \underline{S} E_{2}}\left(x_{1}, z\right)\left(y_{1}, z\right) \leq \min \left\{\mu_{\underline{R} V_{1} \ltimes \underline{R} V_{2}}\left(x_{1}, z\right), \mu_{\underline{R} V_{1} \ltimes \underline{R} V_{2}}\left(y_{1}, z\right)\right\} \text {, } \\
& \sigma_{\underline{S} E_{1} \ltimes \underline{S} E_{2}}\left(x_{1}, z\right)\left(y_{1}, z\right)=\sigma_{\underline{S} E_{1}}\left(x_{1}, y_{1}\right) \wedge \sigma_{\underline{R} V_{2}}(z) \\
& \leq\left(\sigma_{\underline{R} V_{1}}\left(x_{1}\right) \wedge \sigma_{\underline{R} V_{1}}\left(y_{1}\right)\right) \wedge \sigma_{\underline{R} V_{2}}(z) \\
& =\left(\sigma_{\underline{R} V_{1}}\left(x_{1}\right) \wedge \sigma_{\underline{R} V_{2}}(z)\right) \wedge\left(\sigma_{\underline{R} V_{1}}\left(y_{1}\right) \wedge \sigma_{\underline{R} V_{2}}(z)\right) \\
& =\sigma_{\underline{R} V_{1} \ltimes \underline{R} V_{2}}\left(x_{1}, z\right) \wedge \sigma_{\underline{R} V_{1} \ltimes \underline{R} V_{2}}\left(y_{1}, z\right) \\
& \sigma_{\underline{S} E_{1} \ltimes \underline{S} E_{2}}\left(x_{1}, z\right)\left(y_{1}, z\right) \leq \min \left\{\sigma_{\underline{R} V_{1} \ltimes \underline{R} V_{2}}\left(x_{1}, z\right), \sigma_{\underline{R} V_{1} \ltimes \underline{R} V_{2}}\left(y_{1}, z\right)\right\}, \\
& \lambda_{\underline{S} E_{1} \ltimes \underline{S}_{2}}\left(x_{1}, z\right)\left(y_{1}, z\right)=\lambda_{\underline{S} E_{1}}\left(x_{1}, y_{1}\right) \vee \lambda_{\underline{R} V_{2}}(z) \\
& \leq\left(\lambda_{\underline{R} V_{1}}\left(x_{1}\right) \vee \lambda_{\underline{R} V_{1}}\left(y_{1}\right)\right) \vee \lambda_{\underline{R} V_{2}}(z) \\
& =\left(\lambda_{\underline{R} V_{1}}\left(x_{1}\right) \vee \lambda_{\underline{R} V_{2}}(z)\right) \vee\left(\lambda_{\underline{R} V_{1}}\left(y_{1}\right) \vee \lambda_{\underline{R} V_{2}}(z)\right) \\
& =\lambda_{\underline{R} V_{1} \ltimes \underline{R} V_{2}}\left(x_{1}, z\right) \vee \lambda_{\underline{R} V_{1} \ltimes \underline{R} V_{2}}\left(y_{1}, z\right) \\
& \lambda_{\underline{S} E_{1} \ltimes \underline{S} E_{2}}\left(x_{1}, z\right)\left(y_{1}, z\right) \leq \max \left\{\lambda_{\underline{R} V_{1} \ltimes \underline{R} V_{2}}\left(x_{1}, z\right), \lambda_{\underline{R} V_{1} \ltimes \underline{R} V_{2}}\left(y_{1}, z\right)\right\} \text {. }
\end{aligned}
$$

Thus, from above, it is clear that $\underline{S} E_{1} \ltimes \underline{S} E_{2}$ is a neutrosophic relation on $\underline{R} V_{1} \ltimes \underline{R} V_{2}$.

Similarly, we can show that $\bar{S} E_{1} \ltimes \bar{S} E_{2}$ is a neutrosophic relation on $\bar{R} V_{1} \ltimes \bar{R} V_{2}$. Hence, $G=\left(\underline{G}_{1} \ltimes \underline{G}_{2}, \bar{G}_{1} \ltimes \bar{G}_{2}\right)$ is a rough neutrosophic digraph.

Definition 8. The composition of two rough neutrosophic digraphs $G_{1}$ and $G_{2}$ is a rough neutrosophic digraph $G=G_{1} \circ G_{2}=\left(\underline{G}_{1} \circ \underline{G}_{2}, \bar{G}_{1} \circ \bar{G}_{2}\right)$, where $\underline{G}_{1} \circ \underline{G}_{2}=\left(\underline{R} V_{1} \circ \underline{R} V_{2}, \underline{S} E_{1} \circ \underline{S} E_{2}\right)$ and $\bar{G}_{1} \circ \bar{G}_{2}=\left(\bar{R} V_{1} \circ\right.$ $\left.\bar{R} V_{2}, \bar{S} E_{1} \circ \bar{S} E_{2}\right)$ are neutrosophic digraphs, respectively, such that

$$
\mu_{\underline{R} V_{1} \circ \underline{R} V_{2}}\left(x_{1}, x_{2}\right)=\min \left\{\mu_{\underline{R} V_{1}}\left(x_{1}\right), \mu_{\underline{R} V_{2}}\left(x_{2}\right)\right\},
$$




$$
\begin{aligned}
& \sigma_{\underline{R} V_{1} \circ \underline{R} V_{2}}\left(x_{1}, x_{2}\right)=\min \left\{\sigma_{\underline{R} V_{1}}\left(x_{1}\right), \mu_{\underline{R} V_{2}}\left(x_{2}\right)\right\}, \\
& \lambda_{\underline{R} V_{1} \circ \underline{R} V_{2}}\left(x_{1}, x_{2}\right)=\max \left\{\lambda_{\underline{R} V_{1}}\left(x_{1}\right), \mu_{\underline{R} V_{2}}\left(x_{2}\right)\right\} \quad \forall\left(x_{1}, x_{2}\right) \in \underline{R} V_{1} \times \underline{R} V_{2} ， \\
& \mu_{\underline{S} E_{1} \circ \underline{S} E_{2}}\left(x, x_{2}\right)\left(x, y_{2}\right)=\min \left\{\mu_{\underline{R} V_{1}}(x), \mu_{\underline{S} E_{2}}\left(x_{2}, y_{2}\right)\right\} \text {, } \\
& \sigma_{\underline{S} E_{1} \circ \underline{S} E_{2}}\left(x, x_{2}\right)\left(x, y_{2}\right)=\min \left\{\sigma_{\underline{R} V_{1}}(x), \sigma_{\underline{S} E_{2}}\left(x_{2}, y_{2}\right)\right\} \text {, } \\
& \lambda_{\underline{S} E_{1} \circ \underline{S} E_{2}}\left(x, x_{2}\right)\left(x, y_{2}\right)=\max \left\{\lambda_{\underline{R} V_{1}}(x), \lambda_{\underline{S} E_{2}}\left(x_{2}, y_{2}\right)\right\} \quad \forall x \in \underline{R} V_{1}, x_{2} y_{2} \in \underline{S} E_{2} \text {, } \\
& \mu_{\underline{S} E_{1} \circ \underline{S} E_{2}}\left(x_{1}, z\right)\left(y_{1}, z\right)=\min \left\{\mu_{\underline{S} E_{1}}\left(x_{1}, y_{1}\right), \mu_{\underline{R} V_{2}}(z)\right\} \text {, } \\
& \sigma_{\underline{S} E_{1} \circ \underline{S} E_{2}}\left(x_{1}, z\right)\left(y_{1}, z\right)=\min \left\{\sigma_{\underline{S} E_{1}}\left(x_{1}, y_{1}\right), \sigma_{\underline{R} V_{2}}(z)\right\} \text {, } \\
& \lambda_{\underline{S} E_{1} \circ \underline{S} E_{2}}\left(x_{1}, z\right)\left(y_{1}, z\right)=\max \left\{\lambda_{\underline{S} E_{1}}\left(x_{1}, y_{1}\right), \lambda_{\underline{R} V_{2}}(z)\right\} \quad \forall x_{1} y_{1} \in \underline{S} E_{1}, z \in \underline{R} V_{2} \text {, } \\
& \mu_{\underline{S} E_{1} \circ \underline{S} E_{2}}\left(x_{1}, x_{2}\right)\left(y_{1}, y_{2}\right)=\min \left\{\mu_{\underline{S} E_{1}}\left(x_{1}, y_{1}\right), \mu_{\underline{R} V_{2}}\left(x_{2}\right), \mu_{\underline{R} V_{2}}\left(y_{2}\right)\right\} \text {, } \\
& \sigma_{\underline{S} E_{1} \circ \underline{S} E_{2}}\left(x_{1}, x_{2}\right)\left(y_{1}, y_{2}\right)=\min \left\{\sigma_{\underline{S} E_{1}}\left(x_{1}, y_{1}\right), \sigma_{\underline{R} V_{2}}\left(x_{2}\right), \sigma_{\underline{R} V_{2}}\left(y_{2}\right)\right\} \text {, } \\
& \lambda_{\underline{S} E_{1} \circ \underline{S} E_{2}}\left(x_{1}, x_{2}\right)\left(y_{1}, y_{2}\right)=\max \left\{\lambda_{\underline{S} E_{1}}\left(x_{1}, y_{1}\right), \lambda_{\underline{R} V_{2}}\left(x_{2}\right), \lambda_{\underline{R} V_{2}}\left(y_{2}\right)\right\} \\
& \forall x_{1} y_{1} \in \underline{S} E_{1}, x_{2}, y_{2} \in \underline{R} V_{2}, x_{2} \neq y_{2} . \\
& \text { (2) } \mu_{\bar{R} V_{1} \circ \bar{R} V_{2}}\left(x_{1}, x_{2}\right)=\min \left\{\mu_{\bar{R} V_{1}}\left(x_{1}\right), \mu_{\bar{R} V_{2}}\left(x_{2}\right)\right\} \text {, } \\
& \sigma_{\bar{R} V_{1} \circ \bar{R} V_{2}}\left(x_{1}, x_{2}\right)=\min \left\{\sigma_{\bar{R} V_{1}}\left(x_{1}\right), \mu_{\bar{R} V_{2}}\left(x_{2}\right)\right\} \text {, } \\
& \lambda_{\bar{R} V_{1} \circ \bar{R} V_{2}}\left(x_{1}, x_{2}\right)=\max \left\{\lambda_{\bar{R} V_{1}}\left(x_{1}\right), \mu_{\bar{R} V_{2}}\left(x_{2}\right)\right\} \quad \forall\left(x_{1}, x_{2}\right) \in \bar{R} V_{1} \times \bar{R} V_{2}, \\
& \mu_{\bar{S} E_{1} \circ \bar{S} E_{2}}\left(x, x_{2}\right)\left(x, y_{2}\right)=\min \left\{\mu_{\bar{R} V_{1}}(x), \mu_{\bar{S} E_{2}}\left(x_{2}, y_{2}\right)\right\}, \\
& \sigma_{\bar{S} E_{1} \circ \bar{S} E_{2}}\left(x, x_{2}\right)\left(x, y_{2}\right)=\min \left\{\sigma_{\bar{R} V_{1}}(x), \sigma_{\bar{S} E_{2}}\left(x_{2}, y_{2}\right)\right\} \text {, } \\
& \lambda_{\bar{S} E_{1} \circ \bar{S} E_{2}}\left(x, x_{2}\right)\left(x, y_{2}\right)=\max \left\{\lambda_{\bar{R} V_{1}}(x), \lambda_{\bar{S} E_{2}}\left(x_{2}, y_{2}\right)\right\} \quad \forall x \in \bar{R} V_{1}, x_{2} y_{2} \in \bar{S} E_{2} \text {, } \\
& \mu_{\bar{S} E_{1} \circ \bar{S}_{E_{2}}}\left(x_{1}, z\right)\left(y_{1}, z\right)=\min \left\{\mu_{\bar{S} E_{1}}\left(x_{1}, y_{1}\right), \mu_{\bar{R} V_{2}}(z)\right\} \text {, } \\
& \sigma_{\bar{S} E_{1} \circ \bar{S}_{E_{2}}}\left(x_{1}, z\right)\left(y_{1}, z\right)=\min \left\{\sigma_{\bar{S} E_{1}}\left(x_{1}, y_{1}\right), \sigma_{\bar{R} V_{2}}(z)\right\}, \\
& \lambda_{\bar{S} E_{1} \circ \bar{S}_{E_{2}}}\left(x_{1}, z\right)\left(y_{1}, z\right)=\max \left\{\lambda_{\bar{S} E_{1}}\left(x_{1}, y_{1}\right), \lambda_{\bar{R} V_{2}}(z)\right\} \quad \forall x_{1} y_{1} \in \bar{S} E_{1}, z \in \bar{R} V_{2}, \\
& \mu_{\bar{S} E_{1} \circ \bar{S} E_{2}}\left(x_{1}, x_{2}\right)\left(y_{1}, y_{2}\right)=\min \left\{\mu_{\bar{S}_{E_{1}}}\left(x_{1}, y_{1}\right), \mu_{\bar{R} V_{2}}\left(x_{2}\right), \mu_{\bar{R} V_{2}}\left(y_{2}\right)\right\}, \\
& \sigma_{\bar{S} E_{1} \circ \bar{S} E_{2}}\left(x_{1}, x_{2}\right)\left(y_{1}, y_{2}\right)=\min \left\{\sigma_{\bar{S} E_{1}}\left(x_{1}, y_{1}\right), \sigma_{\bar{R} V_{2}}\left(x_{2}\right), \sigma_{\bar{R} V_{2}}\left(y_{2}\right)\right\}, \\
& \lambda_{\bar{S} E_{1} \circ \bar{S} E_{2}}\left(x_{1}, x_{2}\right)\left(y_{1}, y_{2}\right)=\max \left\{\lambda_{\bar{S} E_{1}}\left(x_{1}, y_{1}\right), \lambda_{\bar{R} V_{2}}\left(x_{2}\right), \lambda_{\bar{R} V_{2}}\left(y_{2}\right)\right\} \\
& \forall x_{1} y_{1} \in \bar{S} E_{1}, x_{2}, y_{2} \in \bar{R} V_{2}, x_{2} \neq y_{2}
\end{aligned}
$$

Example 4. Let $V^{*}=\{p, q, r\}$ be a set. Let $G_{1}=\left(\underline{G}_{1}, \bar{G}_{1}\right)$ and $G_{2}=\left(\underline{G}_{2}, \bar{G}_{2}\right)$ be two RND on $V^{*}$, where $\underline{G}_{1}=\left(\underline{R} V_{1}, \underline{S} E_{1}\right)$ and $\bar{G}_{1}=\left(\bar{R} V_{1}, \bar{S} E_{1}\right)$ are $N D$, as shown in Figure 8. $\underline{G}_{2}=\left(\underline{R} V_{2}, \underline{S} E_{2}\right)$ and $\bar{G}_{2}=\left(\bar{R} V_{2}, \bar{S} E_{2}\right)$ are also $N D$, as shown in Figure 9.

The composition of $G_{1}$ and $G_{2}$ is $G=G_{1} \circ G_{2}=\left(\underline{G}_{1} \circ \underline{G}_{2}, \bar{G}_{1} \circ \bar{G}_{2}\right)$ where $\underline{G}_{1} \circ \underline{G}_{2}=\left(\underline{R} V_{1} \circ \underline{R} V_{2}, \underline{S} E_{1} \circ\right.$ $\left.\underline{S} E_{2}\right)$ and $\bar{G}_{1} \circ \bar{G}_{2}=\left(\bar{R} V_{1} \circ \bar{R} V_{2}, \bar{S} E_{1} \circ \bar{S} E_{2}\right)$ are NDs, as shown in Figures 10 and 11 . 


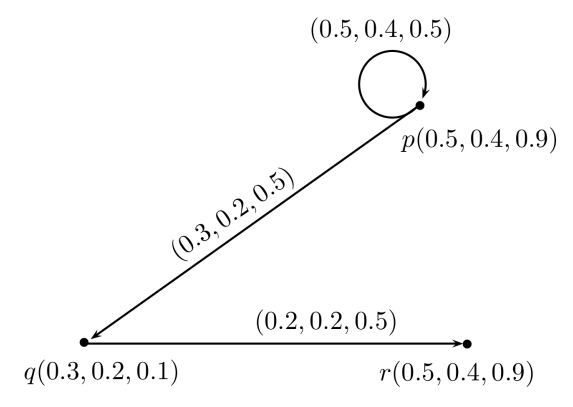

$\underline{G}_{1}=\left(\underline{R} V_{1}, \underline{S} E_{1}\right)$

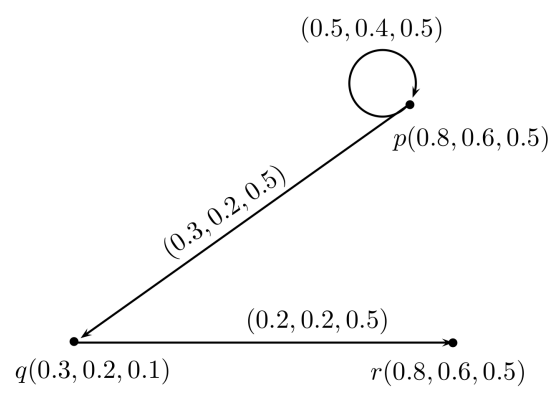

$\bar{G}_{1}=\left(\bar{R} V_{1}, \bar{S} E_{1}\right)$

Figure 8. Rough neutrosophic digraph $G_{1}=\left(\underline{G}_{1}, \bar{G}_{1}\right)$.

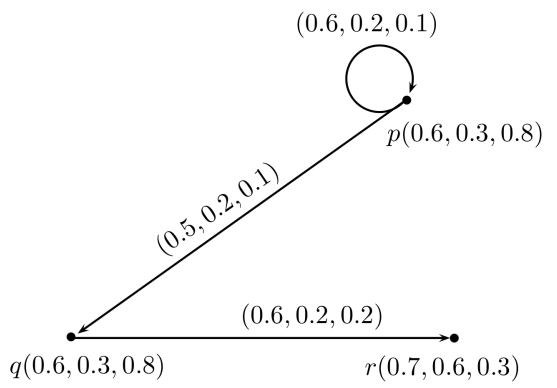

$\underline{G}_{2}=\left(\underline{R} V_{2}, \underline{S} E_{2}\right)$

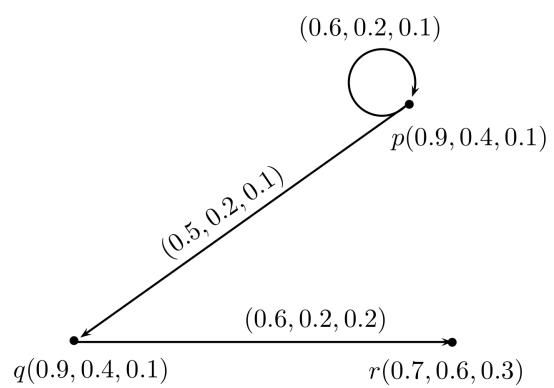

$\bar{G}_{2}=\left(\bar{R} V_{2}, \bar{S} E_{2}\right.$

Figure 9. Rough neutrosophic digraph $G_{2}=\left(\underline{G}_{2}, \bar{G}_{2}\right)$.

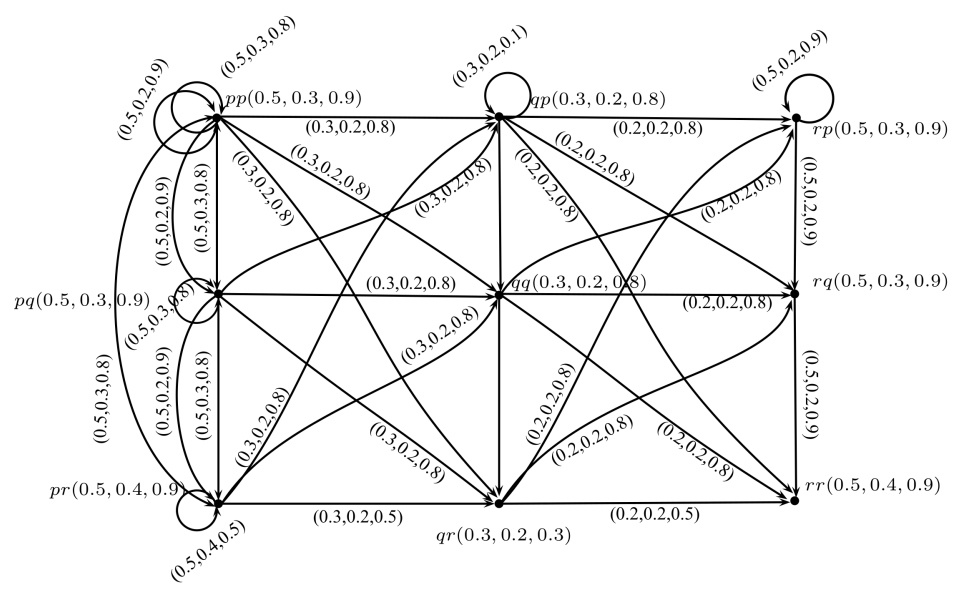

Figure 10. Neutrosophic digraph $\underline{G}_{1} \circ \underline{G}_{2}=\left(\underline{R} V_{1} \circ \underline{R} V_{2}, \underline{S} E_{1} \circ \underline{S} E_{2}\right)$. 


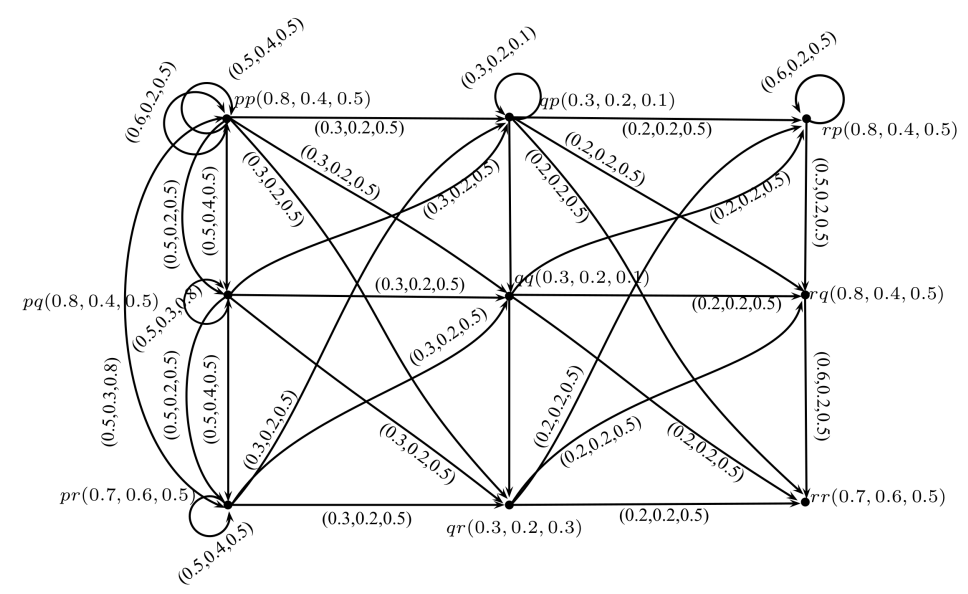

Figure 11. Neutrosophic digraph $\bar{G}_{1} \circ \bar{G}_{2}=\left(\underline{R} V_{1} \circ \underline{R} V_{2}, \underline{S} E_{1} \circ \underline{S} E_{2}\right)$.

Theorem 3. The Composition of two rough neutrosophic digraphs is a rough neutrosophic digraph.

Proof. Let $G_{1}=\left(\underline{G}_{1}, \bar{G}_{1}\right)$ and $G_{2}=\left(\underline{G}_{2}, \bar{G}_{2}\right)$ be two rough neutrosophic digraphs. Let $G=G_{1} \circ G_{2}=$ $\left(\underline{G}_{1} \circ \underline{G}_{2}, \bar{G}_{1} \circ \bar{G}_{2}\right)$ be the Composition of $G_{1}$ and $G_{2}$, where $\underline{G}_{1} \circ \underline{G}_{2}=\left(\underline{R} V_{1} \circ \underline{R} V_{2}, \underline{S} E_{1} \circ \underline{S} E_{2}\right)$ and $\bar{G}_{1} \circ \bar{G}_{2}=\left(\bar{R} V_{1} \circ \bar{R} V_{2}, \bar{S} E_{1} \circ \bar{S} E_{2}\right)$. To prove that $G=\underline{G}_{1} \circ \bar{G}_{2}$ is a rough neutrosophic digraph, it is enough to show that $\underline{S} E_{1} \circ \underline{S} E_{2}$ and $\bar{S} E_{1} \circ \bar{S} E_{2}$ are neutrosophic relations on $\underline{R} V_{1} \circ \underline{R} V_{2}$ and $\bar{R} V_{1} \circ \bar{R} V_{2}$, respectively. First, we show that $\underline{S} E_{1} \circ \underline{S} E_{2}$ is a neutrosophic relation on $\underline{R} V_{1} \circ \underline{R} V_{2}$.

If $x \in \underline{R} V_{1}, x_{2} y_{2} \in \underline{S} E_{2}$, then

$$
\begin{aligned}
& \mu_{\underline{S} E_{1} \circ \underline{S} E_{2}}\left(x, x_{2}\right)\left(x, y_{2}\right)=\mu_{\underline{R} V_{1}}(x) \wedge \mu_{\underline{S} E_{2}}\left(x_{2}, y_{2}\right) \\
& \leq \mu_{\underline{R} V_{1}}(x) \wedge\left(\mu_{\underline{R} V_{2}}\left(x_{2}\right) \wedge \mu_{\underline{R} V_{2}}\left(y_{2}\right)\right) \\
& =\left(\mu_{\underline{R} V_{1}}(x) \wedge \mu_{\underline{R} V_{2}}\left(x_{2}\right)\right) \wedge\left(\mu_{\underline{R} V_{1}}(x) \wedge \mu_{\underline{R} V_{2}}\left(y_{2}\right)\right) \\
& =\mu_{\underline{R} V_{1} \circ \underline{R} V_{2}}\left(x, x_{2}\right) \wedge \mu_{\underline{R} V_{1} \circ \underline{R} V_{2}}\left(x, y_{2}\right) \\
& \mu_{\underline{S} E_{1} \circ \underline{S} E_{2}}\left(x, x_{2}\right)\left(x, y_{2}\right) \leq \min \left\{\mu_{\underline{R} V_{1} \circ \underline{R} V_{2}}\left(x, x_{2}\right), \mu_{\underline{R} V_{1} \circ \underline{R} V_{2}}\left(x, y_{2}\right)\right\} \text {, } \\
& \sigma_{\underline{S} E_{1} \circ \underline{S} E_{2}}\left(x, x_{2}\right)\left(x, y_{2}\right)=\sigma_{\underline{R} V_{1}}(x) \wedge \sigma_{\underline{S}_{2}}\left(x_{2}, y_{2}\right) \\
& \leq \sigma_{\underline{R} V_{1}}(x) \wedge\left(\sigma_{\underline{R} V_{2}}\left(x_{2}\right) \wedge \sigma_{\underline{R} V_{2}}\left(y_{2}\right)\right) \\
& =\left(\sigma_{\underline{R} V_{1}}(x) \wedge \sigma_{\underline{R} V_{2}}\left(x_{2}\right)\right) \wedge\left(\sigma_{\underline{R} V_{1}}(x) \wedge \sigma_{\underline{R} V_{2}}\left(y_{2}\right)\right. \\
& =\sigma_{\underline{R} V_{1} \circ \underline{R} V_{2}}\left(x, x_{2}\right) \wedge \sigma_{\underline{R} V_{1} \circ \underline{R} V_{2}}\left(x, y_{2}\right) \\
& \sigma_{\underline{S} E_{1} \circ \underline{S} E_{2}}\left(x, x_{2}\right)\left(x, y_{2}\right) \leq \min \left\{\sigma_{\underline{R} V_{1} \circ \underline{R} V_{2}}\left(x, x_{2}\right), \sigma_{\underline{R} V_{1} \circ \underline{R} V_{2}}\left(x, y_{2}\right)\right\} \text {, } \\
& \lambda_{\underline{S} E_{1} \circ \underline{S} E_{2}}\left(x, x_{2}\right)\left(x, y_{2}\right)=\lambda_{\underline{R} V_{1}}(x) \vee \lambda_{\underline{S} E_{2}}\left(x_{2}, y_{2}\right) \\
& \leq \lambda_{\underline{R} V_{1}}(x) \vee\left(\lambda_{\underline{R} V_{2}}\left(x_{2}\right) \vee \lambda_{\underline{R} V_{2}}\left(y_{2}\right)\right) \\
& =\left(\lambda_{\underline{R} V_{1}}(x) \vee \lambda_{\underline{R} V_{2}}\left(x_{2}\right)\right) \vee\left(\lambda_{\underline{R} V_{1}}(x) \vee \lambda_{\underline{R} V_{2}}\left(y_{2}\right)\right) \\
& =\lambda_{\underline{R} V_{1} \circ \underline{R} V_{2}}\left(x, x_{2}\right) \vee \lambda_{\underline{R} V_{1} \circ \underline{R} V_{2}}\left(x, y_{2}\right) \\
& \lambda_{\underline{S} E_{1} \circ \underline{S} E_{2}}\left(x, x_{2}, x, y_{2}\right) \leq \max \left\{\lambda_{\underline{R} V_{1} \circ \underline{R} V_{2}}\left(x, x_{2}\right), \lambda_{\underline{R} V_{1} \circ \underline{R} V_{2}}\left(x, y_{2}\right)\right\} \text {. }
\end{aligned}
$$

If $x_{1} y_{1} \in \underline{S} E_{1}, z \in \underline{R} V_{2}$, then

$$
\begin{aligned}
\mu_{\underline{S} E_{1} \circ \underline{S} E_{2}}\left(x_{1}, z\right)\left(y_{1}, z\right) & =\mu_{\underline{S} E_{1}}\left(x_{1}, y_{1}\right) \wedge \mu_{\underline{R} V_{2}}(z) \\
& \leq\left(\mu_{\underline{R} V_{1}}\left(x_{1}\right) \wedge \mu_{\underline{R} V_{1}}\left(y_{1}\right)\right) \wedge \mu_{\underline{R} V_{2}}(z) \\
& =\left(\mu_{\underline{R} V_{1}}\left(x_{1}\right) \wedge \mu_{\underline{R} V_{2}}(z)\right) \wedge\left(\mu_{\underline{R} V_{1}}\left(y_{1}\right) \wedge \mu_{\underline{R} V_{2}}(z)\right) \\
& =\mu_{\underline{R} V_{1} \circ \underline{R} V_{2}}\left(x_{1}, z\right) \wedge \mu_{\underline{R} V_{1} \circ \underline{R} V_{2}}\left(y_{1}, z\right) \\
\mu_{\underline{S} E_{1} \circ \underline{S} E_{2}}\left(x_{1}, z\right)\left(y_{1}, z\right) & \leq \min \left\{\mu_{\underline{R} V_{1} \circ \underline{R} V_{2}}\left(x_{1}, z\right), \mu_{\underline{R} V_{1} \circ \underline{R} V_{2}}\left(y_{1}, z\right)\right\}
\end{aligned}
$$




$$
\begin{aligned}
& \sigma_{\underline{S} E_{1} \circ \underline{S} E_{2}}\left(x_{1}, z\right)\left(y_{1}, z\right)=\sigma_{\underline{S} E_{1}}\left(x_{1}, y_{1}\right) \wedge \sigma_{\underline{R} V_{2}}(z) \\
& \leq\left(\sigma_{\underline{R} V_{1}}\left(x_{1}\right) \wedge \sigma_{\underline{R} V_{1}}\left(y_{1}\right)\right) \wedge \sigma_{\underline{R} V_{2}}(z) \\
& =\left(\sigma_{\underline{R} V_{1}}\left(x_{1}\right) \wedge \sigma_{\underline{R} V_{2}}(z)\right) \wedge\left(\sigma_{\underline{R} V_{1}}\left(y_{1}\right) \wedge \sigma_{\underline{R} V_{2}}(z)\right) \\
& =\sigma_{\underline{R} V_{1} \circ \underline{R} V_{2}}\left(x_{1}, z\right) \wedge \sigma_{\underline{R} V_{1} \circ \underline{R} V_{2}}\left(y_{1}, z\right) \\
& \sigma_{\underline{S} E_{1} \circ \underline{S} E_{2}}\left(x_{1}, z\right)\left(y_{1}, z\right) \leq \min \left\{\sigma_{\underline{R} V_{1} \circ \underline{R} V_{2}}\left(x_{1}, z\right), \sigma_{\underline{R} V_{1} \circ \underline{R} V_{2}}\left(y_{1}, z\right)\right\} \text {, } \\
& \lambda_{\underline{S} E_{1} \circ \underline{S} E_{2}}\left(x_{1}, z\right)\left(y_{1}, z\right)=\lambda_{\underline{S} E_{1}}\left(x_{1}, y_{1}\right) \vee \lambda_{\underline{R} V_{2}}(z) \\
& \leq\left(\lambda_{\underline{R} V_{1}}\left(x_{1}\right) \vee \lambda_{\underline{R} V_{1}}\left(y_{1}\right)\right) \vee \lambda_{\underline{R} V_{2}}(z) \\
& =\left(\lambda_{\underline{R} V_{1}}\left(x_{1}\right) \vee \lambda_{\underline{R} V_{2}}(z)\right) \vee\left(\lambda_{\underline{R} V_{1}}\left(y_{1}\right) \vee \lambda_{\underline{R} V_{2}}(z)\right) \\
& =\lambda_{\underline{R} V_{1} \circ \underline{R} V_{2}}\left(x_{1}, z\right) \vee \lambda_{\underline{R} V_{1} \circ \underline{R} V_{2}}\left(y_{1}, z\right) \\
& \lambda_{\underline{S} E_{1} \circ \underline{S} E_{2}}\left(x_{1}, z\right)\left(y_{1}, z\right) \leq \max \left\{\lambda_{\underline{R} V_{1} \circ \underline{R} V_{2}}\left(x_{1}, z\right), \lambda_{\underline{R} V_{1} \circ \underline{R} V_{2}}\left(y_{1}, z\right)\right\} \text {. }
\end{aligned}
$$

If $x_{1} y_{1} \in \underline{S} E_{1}, x_{2}, y_{2} \in \underline{R} V_{2}$ such that $x_{2} \neq y_{2}$,

$$
\begin{aligned}
& \mu_{\underline{S} E_{1} \circ \underline{S} E_{2}}\left(x_{1}, x_{2}\right)\left(y_{1}, y_{2}\right)=\mu_{\underline{S} E_{1}}\left(x_{1} y_{1}\right) \wedge \mu_{\underline{R} V_{2}}\left(x_{2}\right) \wedge \mu_{\underline{R} V_{2}}\left(y_{2}\right) \\
& \leq\left(\mu_{\underline{R} V_{1}}\left(x_{1}\right) \wedge \mu_{\underline{R} V_{1}}\left(y_{1}\right)\right) \wedge \mu_{\underline{R} V_{2}}\left(x_{2}\right) \wedge \mu_{\underline{R} V_{2}}\left(y_{2}\right) \\
& \left.=\left(\mu_{\underline{R} V_{1}}\left(x_{1}\right) \wedge \mu_{\underline{R} V_{2}}\left(x_{2}\right)\right) \wedge\left(\mu_{\underline{R} V_{1}}\left(y_{1}\right)\right) \wedge \mu_{\underline{R} V_{2}}\left(y_{2}\right)\right) \\
& =\mu_{\underline{R} V_{1} \circ \underline{R} V_{2}}\left(x_{1}, x_{2}\right) \wedge \mu_{\underline{R} V_{1} \circ \underline{R} V_{2}}\left(y_{1}, y_{2}\right) \\
& \mu_{\underline{S} E_{1} \circ \underline{S} E_{2}}\left(x_{1}, x_{2}\right)\left(y_{1}, y_{2}\right) \leq \min \left\{\mu_{\underline{R} V_{1} \circ \underline{R} V_{2}}\left(x_{1}, x_{2}\right), \mu_{\underline{R} V_{1} \circ \underline{R} V_{2}}\left(y_{1}, y_{2}\right)\right\} \\
& \sigma_{\underline{S} E_{1} \circ \underline{S} E_{2}}\left(x_{1}, x_{2}\right)\left(y_{1}, y_{2}\right)=\sigma_{\underline{S} E_{1}}\left(x_{1} y_{1}\right) \wedge \sigma_{\underline{R} V_{2}}\left(x_{2}\right) \wedge \sigma_{\underline{R} V_{2}}\left(y_{2}\right) \\
& \leq\left(\sigma_{\underline{R} V_{1}}\left(x_{1}\right) \wedge \sigma_{\underline{R} V_{1}}\left(y_{1}\right)\right) \wedge \sigma_{\underline{R} V_{2}}\left(x_{2}\right) \wedge \sigma_{\underline{R} V_{2}}\left(y_{2}\right) \\
& \left.=\left(\sigma_{\underline{R} V_{1}}\left(x_{1}\right) \wedge \sigma_{\underline{R} V_{2}}\left(x_{2}\right)\right) \wedge\left(\sigma_{\underline{R} V_{1}}\left(y_{1}\right)\right) \wedge \sigma_{\underline{R} V_{2}}\left(y_{2}\right)\right) \\
& =\sigma_{\underline{R} V_{1} \circ \underline{R} V_{2}}\left(x_{1}, x_{2}\right) \wedge \sigma_{\underline{R} V_{1} \circ \underline{R} V_{2}}\left(y_{1}, y_{2}\right) \\
& \sigma_{\underline{S} E_{1} \circ \underline{S} E_{2}}\left(x_{1}, x_{2}\right)\left(y_{1}, y_{2}\right) \leq \min \left\{\sigma_{\underline{R} V_{1} \circ \underline{R} V_{2}}\left(x_{1}, x_{2}\right), \sigma_{\underline{R} V_{1} \circ \underline{R} V_{2}}\left(y_{1}, y_{2}\right)\right\} \\
& \lambda_{\underline{S} E_{1} \circ \underline{S E}_{2}}\left(x_{1}, x_{2}\right)\left(y_{1}, y_{2}\right)=\lambda_{\underline{S} E_{1}}\left(x_{1} y_{1}\right) \vee \lambda_{\underline{R} V_{2}}\left(x_{2}\right) \vee \lambda_{\underline{R} V_{2}}\left(y_{2}\right) \\
& \leq\left(\lambda_{\underline{R} V_{1}}\left(x_{1}\right) \vee \lambda_{\underline{R} V_{1}}\left(y_{1}\right)\right) \vee \lambda_{\underline{R} V_{2}}\left(x_{2}\right) \vee \lambda_{\underline{R} V_{2}}\left(y_{2}\right) \\
& \left.=\left(\lambda_{\underline{R} V_{1}}\left(x_{1}\right) \vee \lambda_{\underline{R} V_{2}}\left(x_{2}\right)\right) \vee\left(\lambda_{\underline{R} V_{1}}\left(y_{1}\right)\right) \vee \lambda_{\underline{R} V_{2}}\left(y_{2}\right)\right) \\
& =\lambda_{\underline{R} V_{1} \circ \underline{R} V_{2}}\left(x_{1}, x_{2}\right) \vee \lambda_{\underline{R} V_{1} \circ \underline{R} V_{2}}\left(y_{1}, y_{2}\right) \\
& \lambda_{\underline{S} E_{1} \circ \underline{S} E_{2}}\left(x_{1}, x_{2}\right)\left(y_{1}, y_{2}\right) \leq \max \left\{\lambda_{\underline{R} V_{1} \circ \underline{R} V_{2}}\left(x_{1}, x_{2}\right), \lambda_{\underline{R} V_{1} \circ \underline{R} V_{2}}\left(y_{1}, y_{2}\right)\right\} \text {. }
\end{aligned}
$$

Thus, from above, it is clear that $\underline{S} E_{1} \circ \underline{S} E_{2}$ is a neutrosophic relation on $\underline{R} V_{1} \circ \underline{R} V_{2}$.

Similarly, we can show that $\bar{S} E_{1} \circ \bar{S} E_{2}$ is a neutrosophic relation on $\bar{R} V_{1} \circ \bar{R} V_{2}$. Hence, $G=\left(\underline{G}_{1} \circ \underline{G}_{2}, \bar{G}_{1} \circ \bar{G}_{2}\right)$ is a rough neutrosophic digraph.

Definition 9. Let $G=(\underline{G}, \bar{G})$ be a RND. The complement of $G$, denoted by $G^{\prime}=\left(\underline{G}^{\prime}, \bar{G}^{\prime}\right)$ is a rough neutrosophic digraph, where $\underline{G}^{\prime}=\left((\underline{R} V)^{\prime},(\underline{S} E)^{\prime}\right)$ and $\bar{G}^{\prime}=\left((\bar{R} V)^{\prime},(\bar{S} E)^{\prime}\right)$ are neutrosophic digraph such that

$(\mathbf{1})$

$$
\begin{aligned}
\mu_{(\underline{R} V)^{\prime}}(x) & =\mu_{\underline{R} V}(x), \\
\sigma_{(\underline{R} V)^{\prime}}(x) & =\sigma_{\underline{R} V}(x), \\
\lambda_{(\underline{R} V)^{\prime}}(x) & =\lambda_{\underline{R} V}(x) \forall x \in V^{*} \\
\mu_{(\underline{S} E)^{\prime}}(x, y) & =\min \left\{\mu_{\underline{R} V}(x), \mu_{\underline{R} V}(y)\right\}-\mu_{\underline{S} E}(x y) \\
\sigma_{(\underline{S} E)^{\prime}}(x, y) & =\min \left\{\sigma_{\underline{R} V}(x), \sigma_{\underline{R} V}(y)\right\}-\sigma_{\underline{S} E}(x y) \\
\lambda_{(\underline{S} E)^{\prime}}(x, y) & =\max \left\{\lambda_{\underline{R} V}(x), \lambda_{\underline{R} V}(y)\right\}-\lambda_{\underline{S} E}(x y) \forall x, y \in V^{*} .
\end{aligned}
$$


(2) $\quad \mu_{\bar{R} V^{\prime}}(x)=\mu_{\bar{R} V}(x)$,

$$
\begin{aligned}
\sigma_{\bar{R} V^{\prime}}(x) & =\sigma_{\bar{R} V}(x), \\
\lambda_{\bar{R} V^{\prime}}(x) & =\lambda_{\bar{R} V}(x), \forall x \in V^{*} \\
\mu_{(\bar{S} E)^{\prime}}(x, y) & =\min \left\{\mu_{\bar{R} V}(x), \mu_{\bar{R} V}(y)\right\}-\mu_{\bar{S} E}(x y) \\
\sigma_{(\bar{S} E)^{\prime}}(x, y) & =\min \left\{\sigma_{\bar{R} V}(x), \sigma_{\bar{R} V}(y)\right\}-\sigma_{\bar{S} E}(x y) \\
\lambda_{(\bar{S} E)^{\prime}}(x, y) & =\max \left\{\lambda_{\bar{R} V}(x), \lambda_{\bar{R} V}(y)\right\}-\lambda_{\bar{S} E}(x y) \forall x, y \in V^{*} .
\end{aligned}
$$

Example 5. Consider a rough neutrosophic digraph as shown in Figure 4. The lower and upper approximations of graph $G$ are $\underline{G}=(\underline{R} V, \underline{S E})$ and $\bar{G}=(\bar{R} V, \bar{S} E)$, respectively, where

$$
\begin{gathered}
\underline{R} V=\{(a, 0.2,0.4,0.6),(b, 0.2,0.4,0.6),(c, 0.2,0.5,0.9),(d, 0.2,0.5,0.9)\}, \\
\bar{R} V=\{(a, 0.3,0.8,0.3) .(b, 0.3,0.8,0.3),(c, 0.5,0.6,0.8),(d, 0.5,0.6,0.8)\}, \\
\underline{S} E=\{(a a, 0.2,0.3,0.3),(a b, 0.2,0.3,0.3),(a d, 0.1,0.3,0.8),(b c, 0.1,0.3,0.8), \\
(b d, 0.1,0.3,0.8),(d c, 0.2,0.4,0.7),(d d, 0.2,0.4,0.7)\}, \\
\bar{S} E=\{(a a, 0.2,0.4,0.3),(a b, 0.2,0.4,0.3),(a d, 0.2,0.4,0.7),(b c, 0.2,0.4,0.7), \\
(b d, 0.2,0.4,0.7),(d c, 0.2,0.4,0.7),(d d, 0.2,0.4,0.7)\}
\end{gathered}
$$

The complement of $G$ is $G^{\prime}=\left(\underline{G}^{\prime}, \bar{G}^{\prime}\right)$. By calculations, we have

$$
\begin{aligned}
& (\underline{R} V)^{\prime}=\{(a, 0.2,0.4,0.6),(b, 0.2,0.4,0.6),(c, 0.2,0.5,0.9),(d, 0.2,0.5,0.9)\}, \\
& (\bar{R} V)^{\prime}=\{(a, 0.3,0.8,0.3) .(b, 0.3,0.8,0.3),(c, 0.5,0.6,0.8),(d, 0.5,0.6,0.8)\},
\end{aligned}
$$

$(\underline{S} E)^{\prime}=\{(a a, 0,0.1,0.3),(a b, 0,0.1,0.3),(a c, 0.2,0.4,0.9),(a d, 0.1,0.1,0.1),(b a, 0.2,0.4,0.6),(b b, 0.2,0.4,0.6)$,

$(b c, 0.1,0.1,0.1),(b d, 0.1,0.1,0.1),(c a, 0.2,0.4,0.9),(c b, 0.2,0.4,0.9),(c c, 0.2,0.5,0.9),(c d, 0.2,0.5,0.9)$,

$(d a, 0.2,0.4,0.9),(d b, 0.2,0.4,0.9),(d c, 0,0.1,0.2),(d d, 0,0.1,0.2)\}$,

$(\bar{S} E)^{\prime}=\{(a a, 0.1,0.4,0),(a b, 0.1,0.4,0),(a c, 0.3,0.6,0.8),(a d, 0.1,0.2,0.1),(b a, 0.3,0.8,0.3),(b b, 0.3,0.8,0.3)$,

$(b c, 0.1,0.2,0.1),(b d, 0.1,0.2,0.1),(c a, 0.3,0.6,0.8),(c b, 0.3,0.6,0.8),(c c, 0.5,0.6,0.8),(c d, 0.5,0.6,0.8)$,

$(d a, 0.3,0.6,0.8),(d b, 0.3,0.6,0.8),(d c, 0.3,0.2,0.1),(d d, 0.3,0.2,0.1)\}$.

Thus, $\underline{G}^{\prime}=\left((\underline{R} V)^{\prime},(\underline{S} E)^{\prime}\right)$ and $\bar{G}^{\prime}=\left((\bar{R} V)^{\prime},(\bar{S} E)^{\prime}\right)$ are neutrosophic digraph, as shown in Figure 12.

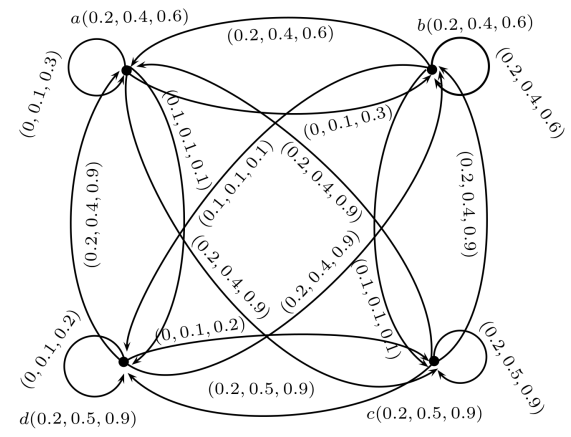

$\underline{G}^{\prime}=\left((\underline{R} N)^{\prime},(\underline{S} E)^{\prime}\right)$

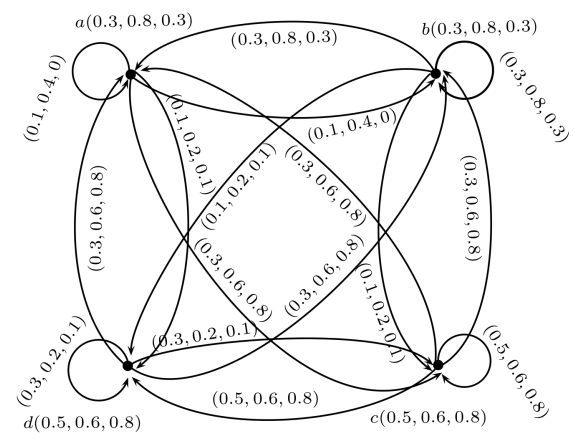

$\bar{G}^{\prime}=\left((\bar{R} N)^{\prime},(\bar{S} E)^{\prime}\right)$

Figure 12. Rough neutrosophic digraph $G^{\prime}=\left(\underline{G}^{\prime}, \bar{G}^{\prime}\right)$.

Definition 10. A rough neutrosophic digraph $G=(\underline{G}, \bar{G})$ is self complementary if $G$ and $G^{\prime}$ are isomorphic, that is, $\underline{G} \cong \underline{G}^{\prime}$ and $\bar{G} \cong \bar{G}^{\prime}$. 
Example 6. Let $V^{*}=\{a, b, c\}$ be $a$ set and $R$ an equivalence relation on $V^{*}$ defined as:

$$
R=\left[\begin{array}{lll}
1 & 0 & 1 \\
0 & 1 & 0 \\
1 & 0 & 1
\end{array}\right]
$$

Let $V=\{(a, 0.2,0.4,0.8),(b, 0.2,0.4,0.8),(c, 0.4,0.6,0.4)\}$ be a neutrosophic set on $V^{*}$. The lower and upper approximations of $V$ are given as,

$\underline{R} V=\{(a, 0.2,0.4,0.8),(b, 0.2,0.4,0.8),(c, 0.2,0.4,0.8)\}$,

$\bar{R} V=\{(a, 0.4,0.6,0.4),(b, 0.2,0.4,0.8),(c, 0.4,0.6,0.4)\}$.

Let $E^{*}=\{a a, a b, a c, b a\} \subseteq V^{*} \times V^{*}$ and $S$ an equivalence relation on $E^{*}$ defined as

$$
S=\left[\begin{array}{llll}
1 & 0 & 1 & 0 \\
0 & 1 & 0 & 0 \\
1 & 0 & 1 & 0 \\
0 & 0 & 0 & 1
\end{array}\right]
$$

Let $E=\{(a a, 0.1,0.3,0.2),(a b, 0.1,0.2,0.4),(a c, 0.2,0.2,0.4),(b a, 0.1,0.2,0.4)\}$ be a neutrosophic set on $E^{*}$ and $S E=(\underline{S} E, \bar{S} E)$ a RNR where $\underline{S} E$ and $\bar{S} E$ are given as

$\underline{S} E=\{(a a, 0.1,0.2,0.4),(a b, 0.1,0.2,0.4),(a c, 0.1,0.2,0.4),(b a, 0.1,0.2,0.4)\}$,

$\overline{\bar{S}} E=\{(a a, 0.2,0.3,0.2),(a b, 0.1,0.2,0.4),(a c, 0.2,0.3,0.2),(b a, 0.1,0.2,0.4)\}$.

Thus, $\underline{G}=(\underline{R} V, \underline{S} E)$ and $\bar{G}=(\bar{R} V, \bar{S} E)$ are neutrosophic digraphs, as shown in Figure 13. The complement of $G$ is $G^{\prime}=\left(\underline{G}^{\prime}, \bar{G}^{\prime}\right)$, where $\underline{G}^{\prime}=\underline{G}$ and $\bar{G}^{\prime}=\bar{G}$ are neutrosophic digraphs, as shown in Figure 13, and it can be easily shown that $G$ and $G^{\prime}$ are isomorphic. Hence, $G=(\underline{G}, \bar{G})$ is a self complementary RND.
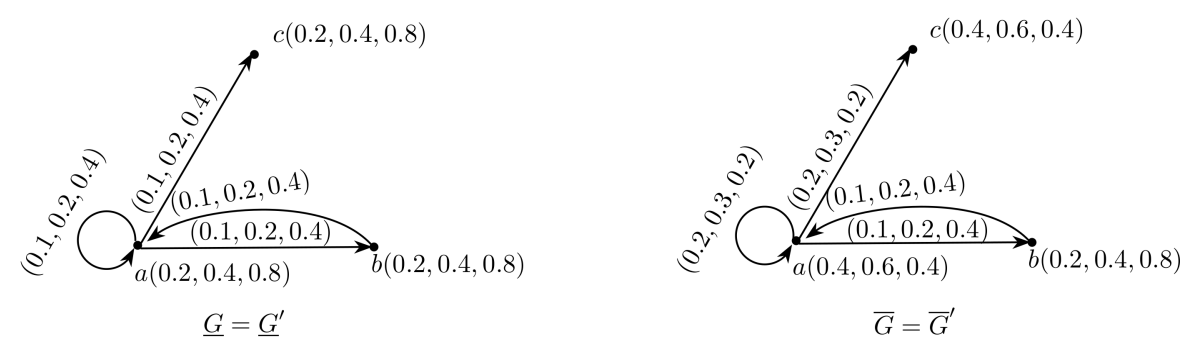

Figure 13. Self complementary RND $G=(\underline{G}, \bar{G})$.

Theorem 4. Let $G=(\underline{G}, \bar{G})$ be a self complementary rough neutrosophic digraph. Then,

$$
\begin{aligned}
\sum_{w, z \in V^{*}} \mu_{\underline{S} E}(w z) & =\frac{1}{2} \sum_{w, z \in V^{*}}\left(\mu_{\underline{R} V}(w) \wedge \mu_{\underline{R} V}(z)\right) \\
\sum_{w, z \in V^{*}} \sigma_{\underline{S} E}(w z) & =\frac{1}{2} \sum_{w, z \in V^{*}}\left(\sigma_{\underline{R} V}(w) \wedge \sigma_{\underline{R} V}(z)\right) \\
\sum_{w, z \in V^{*}} \lambda_{\underline{S} E}(w z) & =\frac{1}{2} \sum_{w, z \in V^{*}}\left(\lambda_{\underline{R} V}(w) \vee \lambda_{\underline{R} V}(z)\right) \\
\sum_{w, z \in V^{*}} \mu_{\bar{S} E}(w z) & =\frac{1}{2} \sum_{w, z \in V^{*}}\left(\mu_{\bar{R} V}(w) \wedge \mu_{\bar{R} V}(z)\right) \\
\sum_{w, z \in V^{*}} \sigma_{\bar{S} E}(w z) & =\frac{1}{2} \sum_{w, z \in V^{*}}\left(\sigma_{\bar{R} V}(w) \wedge \sigma_{\bar{R} V}(z)\right) \\
\sum_{w, z \in V^{*}} \lambda_{\bar{S} E}(w z) & =\frac{1}{2} \sum_{w, z \in V^{*}}\left(\lambda_{\bar{R} V}(w) \vee \lambda_{\bar{R} V}(z)\right) .
\end{aligned}
$$


Proof. Let $G=(\underline{G}, \bar{G})$ be a self complementary rough neutrosophic digraph. Then, there exist two isomorphisms $\underline{g}: V^{*} \longrightarrow V^{*}$ and $\bar{g}: V^{*} \longrightarrow V^{*}$, respectively, such that

$$
\begin{aligned}
\mu_{(\underline{R} V)^{\prime}}(\underline{g}(w)) & =\mu_{\underline{R} V}(w), \\
\left.\sigma_{(\underline{R} V)^{\prime}} \underline{g}(w)\right) & =\sigma_{\underline{R} V}(w), \\
\lambda_{(\underline{R} V)^{\prime}}(\underline{g}(w)) & =\lambda_{\underline{R} V}(w), \quad \forall w \in V^{*} \\
\mu_{(\underline{S} E)^{\prime}}(\underline{g}(w) \underline{g}(z)) & =\mu_{(\underline{S} E)}(w z), \\
\sigma_{(\underline{S} E)^{\prime}}(\underline{g}(w) \underline{g}(z)) & =\sigma_{(\underline{S} E)}(w z), \\
\lambda_{(\underline{S} E)^{\prime}}(\underline{g}(w) \underline{g}(z)) & =\lambda_{(\underline{S} E)}(w z) \forall w, z \in V^{*} .
\end{aligned}
$$

and

$$
\begin{aligned}
\mu_{(\bar{R} V)^{\prime}}(\bar{g}(w)) & =\mu_{\bar{R} V}(w), \\
\sigma_{(\bar{R} V)^{\prime}}(\bar{g}(w)) & =\sigma_{\bar{R} V}(w), \\
\lambda_{(\bar{R} V)^{\prime}}(\bar{g}(w)) & =\lambda_{\bar{R} V}(w), \quad \forall w \in V^{*} \\
\mu_{(\bar{S} E)^{\prime}}(\bar{g}(w) \bar{g}(z)) & =\mu_{(\bar{S} E)}(w z), \\
\sigma_{(\bar{S} E)^{\prime}}(\bar{g}(w) \bar{g}(z)) & =\sigma_{(\bar{S} E)}(w z), \\
\lambda_{(\bar{S} E)^{\prime}}(\bar{g}(w) \bar{g}(z)) & =\lambda_{(\bar{S} E)}(w z) \forall w, z \in V^{*} .
\end{aligned}
$$

By Definition 7, we have

$$
\begin{aligned}
& \mu_{(\underline{S} E)^{\prime}}(\underline{g}(w) \underline{g}(z))=\left(\mu_{\underline{R} V}(w) \wedge \mu_{\underline{R} V}(z)\right)-\mu_{(\underline{S} E)}(w z) \\
& \mu_{(\underline{S} E)}(w z)=\left(\mu_{\underline{R} V}(w) \wedge \mu_{\underline{R} V}(z)\right)-\mu_{(\underline{S} E)}(w z) \\
& \sum_{w, z \in V^{*}} \mu_{(\underline{S} E)}(w z)=\sum_{w, z \in V^{*}}\left(\mu_{\underline{R} V}(w) \wedge \mu_{\underline{R} V}(z)\right)-\sum_{w, z \in V^{*}} \mu_{(\underline{S} E)}(w z) \\
& 2 \sum_{w, z \in V^{*}} \mu_{(\underline{S} E)}(w z)=\sum_{w, z \in V^{*}}\left(\mu_{\underline{R} V}(w) \wedge \mu_{\underline{R} V}(z)\right) \\
& \sum_{w, z \in V^{*}} \mu_{(\underline{S} E)}(w z)=\frac{1}{2} \sum_{w, z \in V^{*}}\left(\mu_{\underline{R} V}(w) \wedge \mu_{\underline{R} V}(z)\right) \\
& \sigma_{(\underline{S} E)^{\prime}}(\underline{g}(w) \underline{g}(z))=\left(\sigma_{\underline{R} V}(w) \wedge \sigma_{\underline{R} V}(z)\right)-\sigma_{(\underline{S} E)}(w z) \\
& \sigma_{(\underline{S} E)}(w z)=\left(\sigma_{\underline{R} V}(w) \wedge \sigma_{\underline{R} V}(z)\right)-\sigma_{(\underline{S} E)}(w z) \\
& \sum_{w, z \in V^{*}} \sigma_{(\underline{S} E)}(w z)=\sum_{w, z \in V^{*}}\left(\sigma_{\underline{R} V}(w) \wedge \sigma_{\underline{R} V}(z)\right)-\sum_{w, z \in V^{*}} \sigma_{(\underline{S} E)}(w z) \\
& 2 \sum_{w, z \in V^{*}} \sigma_{(\underline{S} E)}(w z)=\sum_{w, z \in V^{*}}\left(\sigma_{\underline{R} V}(w) \wedge \sigma_{\underline{R} V}(z)\right) \\
& \sum_{w, z \in V^{*}} \sigma_{(\underline{S} E)}(w z)=\frac{1}{2} \sum_{w, z \in V^{*}}\left(\sigma_{\underline{R} V}(w) \wedge \sigma_{\underline{R} V}(z)\right) \\
& \lambda_{(\underline{S} E)^{\prime}}(\underline{g}(w) \underline{g}(z))=\left(\lambda_{\underline{R} V}(w) \vee \lambda_{\underline{R} V}(z)\right)-\lambda_{(\underline{S} E)}(w z) \\
& \lambda_{(\underline{S} E)}(w z)=\left(\lambda_{\underline{R} V}(w) \vee \lambda_{\underline{R} V}(z)\right)-\lambda_{(\underline{S} E)}(w z) \\
& \sum_{w, z \in V^{*}} \lambda_{(\underline{S} E)}(w z)=\sum_{w, z \in V^{*}}\left(\lambda_{\underline{R} V}(w) \vee \lambda_{\underline{R} V}(z)\right)-\sum_{w, z \in V^{*}} \lambda_{(\underline{S} E)}(w z) \\
& 2 \sum_{w, z \in V^{*}} \lambda_{(\underline{S} E)}(w z)=\sum_{w, z \in V^{*}}\left(\lambda_{\underline{R} V}(w) \vee \lambda_{\underline{R} V}(z)\right) \\
& \sum_{w, z \in V^{*}} \lambda_{(\underline{S} E)}(w z)=\frac{1}{2} \sum_{w, z \in V^{*}}\left(\lambda_{\underline{R} V}(w) \vee \lambda_{\underline{R} V}(z)\right)
\end{aligned}
$$


Similarly, it can be shown that

$$
\begin{aligned}
\sum_{w, z \in V^{*}} \mu_{\bar{S} E}(w z) & =\frac{1}{2} \sum_{w, z \in V^{*}}\left(\mu_{\bar{R} V}(w) \wedge \mu_{\bar{R} V}(z)\right) \\
\sum_{w, z \in V^{*}} \sigma_{\bar{S} E}(w z) & =\frac{1}{2} \sum_{w, z \in V^{*}}\left(\sigma_{\bar{R} V}(w) \wedge \sigma_{\bar{R} V}(z)\right) \\
\sum_{w, z \in V^{*}} \lambda_{\bar{S} E}(w z) & =\frac{1}{2} \sum_{w, z \in V^{*}}\left(\lambda_{\bar{R} V}(w) \vee \lambda_{\bar{R} V}(z)\right) .
\end{aligned}
$$

This completes the proof.

\section{Application}

Investment is a very good way of getting profit and wisely invested money surely gives certain profit. The most important factors that influence individual investment decision are: company's reputation, corporate earnings and price per share. In this application, we combine these factors into one factor, i.e. company's status in industry, to describe overall performance of the company. Let us consider an individual Mr. Shahid who wants to invest his money. For this purpose, he considers some private companies, which are Telecommunication company (TC), Carpenter company (CC), Real Estate business $(R E)$, Vehicle Leasing company $(V L)$, Advertising company $(A D)$, and Textile Testing company $(T T)$. Let $V^{*}=\{T C, C C, R E, V L, A D, T T\}$ be a set. Let $T$ be an equivalence relation defined on $V^{*}$ as follows:

$$
T=\left[\begin{array}{llllll}
1 & 0 & 1 & 0 & 1 & 0 \\
0 & 1 & 0 & 0 & 0 & 0 \\
1 & 0 & 1 & 0 & 1 & 0 \\
0 & 0 & 0 & 1 & 0 & 1 \\
1 & 0 & 1 & 0 & 1 & 0 \\
0 & 0 & 0 & 1 & 0 & 1
\end{array}\right] .
$$

Let $V=\{(T C, 0.3,0.4,0.1),(C C, 0.8,0.1,0.5),(R E, 0.1,0.2,0.6),(V L, 0.9,0.6,0.1),(A D, 0.2,0.5$, $0.2),(T T, 0.8,0.6,0.5)\}$ be a neutrosophic set on $V^{*}$ with three components corresponding to each company, which represents its status in the industry and $T V=(\underline{T} V, \bar{T} V)$ a rough neutrosophic set, where $\underline{T} V$ and $\bar{T} V$ are lower and upper approximations of $V$, respectively, as follows:

$$
\begin{aligned}
\underline{T V}= & \{(T C, 0.1,0.2,0.6),(C C, 0.8,0.1,0.5),(R E, 0.1,0.2,0.6),(V L, 0.8,0.6,0.5),(A D, \\
& 0.1,0.2,0.6),(T T, 0.8,0.6,0.5)\}, \\
\bar{T} V= & \{(T C, 0.3,0.5,0.1),(C C, 0.8,0.1,0.5),(R E, 0.3,0.5,0.1),(V L, 0.9,0.6,0.1),(A D, \\
& 0.3,0.5,0.1),(T T, 0.9,0.6,0.1)\} . \\
\text { Let } E^{*}= & \{(T C, C C),(T C, A D),(T C, R E),(C C, V L),(C C, T T),(A D, R E),(T T, V L)\},
\end{aligned}
$$

be the set of edges and $S$ an equivalence relation on $E^{*}$ defined as follows:

$$
S=\left[\begin{array}{lllllll}
1 & 0 & 0 & 0 & 0 & 0 & 0 \\
0 & 1 & 1 & 0 & 0 & 1 & 0 \\
0 & 1 & 1 & 0 & 0 & 1 & 0 \\
0 & 0 & 0 & 1 & 1 & 0 & 0 \\
0 & 1 & 0 & 1 & 1 & 0 & 0 \\
0 & 0 & 1 & 0 & 0 & 1 & 0 \\
0 & 0 & 0 & 0 & 0 & 0 & 1
\end{array}\right]
$$




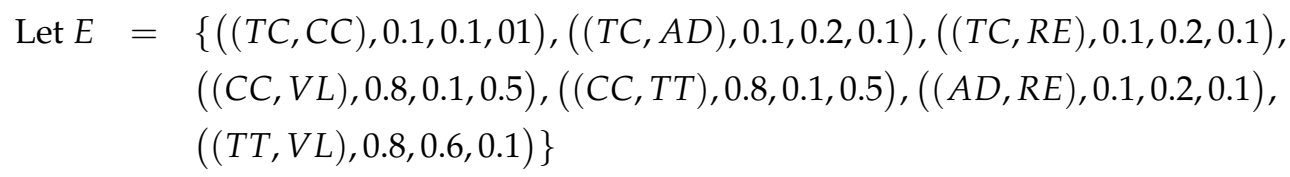

be a neutrosophic set on $E^{*}$ which represents relationship between companies and $S E=(\underline{S} E, \bar{S} E)$ a rough neutrosophic relation, where $\underline{S} E$ and $\bar{S} E$ are lower and upper upper approximations of $E$, respectively, as follows:

$$
\begin{aligned}
& \underline{S} E=\quad\{((T C, C C), 0.1,0.1,0.1),((T C, A D), 0.1,0.2,0.1),((T C, R E), 0.1,0.2,0.1), \\
&((C C, V L), 0.8,0.1,0.5),((C C, T T), 0.8,0.1,0.5),((A D, R E), 0.1,0.2,0.1), \\
&((T T, V L), 0.8,0.6,0.1)\}, \\
& \bar{S} E=\{((T C, C C), 0.1,0.1,0.1),((T C, A D), 0.1,0.2,0.1),((T C, R E), 0.1,0.2,0.1), \\
&((C C, V L), 0.8,0.1,0.5),((C C, T T), 0.8,0.1,0.5),((A D, R E) 0.1,0.2,0.1), \\
&((T T, V L), 0.8,0.6,0.1)\} .
\end{aligned}
$$

Thus, $\underline{G}=(\underline{T} V, \underline{S} E)$ and $\bar{G}=(\bar{T} V, \bar{S} E)$ is a rough neutrosophic digraph as shown in Figure 14 .

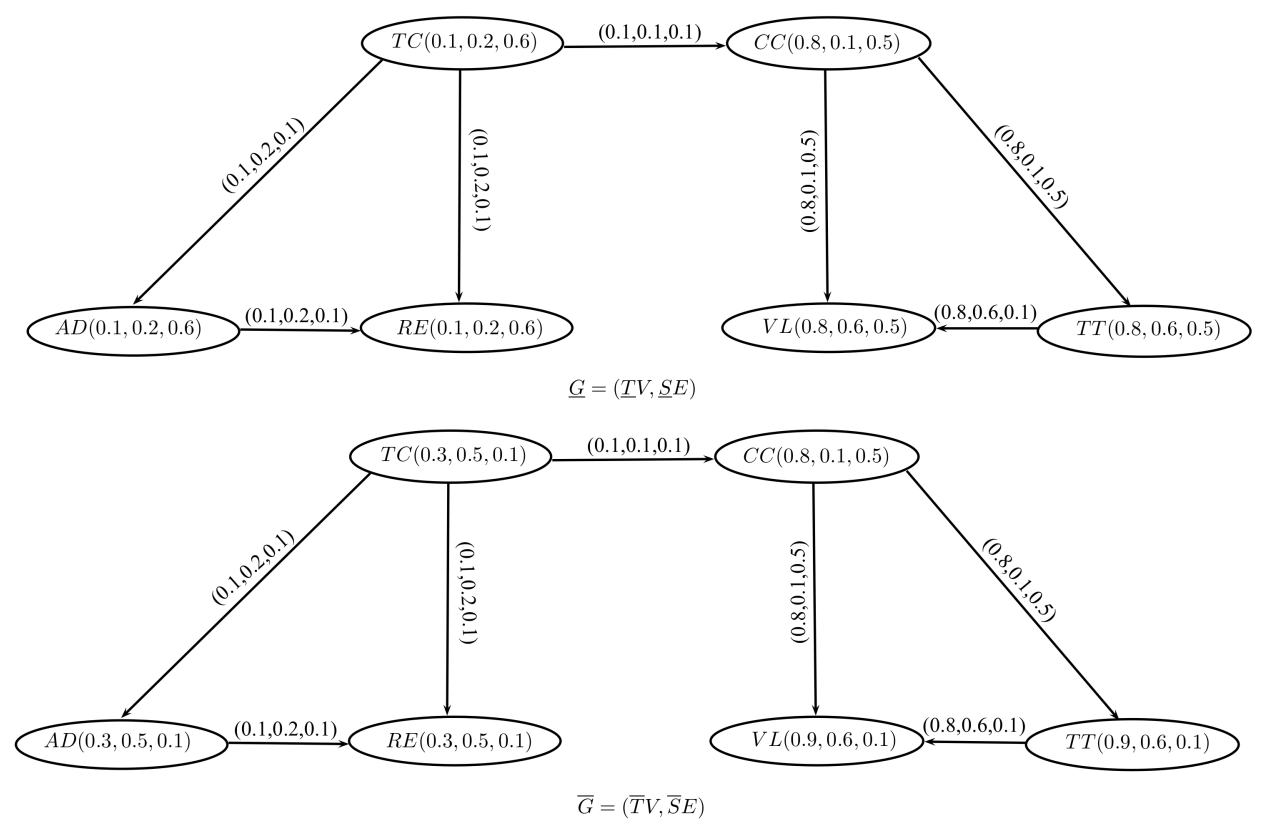

Figure 14. Rough neutrosophic digraph $G=(\underline{G}, \bar{G})$.

To find out the most suitable investment company, we define the score values

$$
S\left(v_{i}\right)=\sum_{v_{i} v_{j} \in E^{*}} \frac{T\left(v_{j}\right)+I\left(v_{j}\right)-F\left(v_{j}\right)}{3-\left(T\left(v_{i} v_{j}\right)+I\left(v_{i} v_{j}\right)-F\left(v_{i} v_{j}\right)\right)},
$$

where

$$
\begin{aligned}
T\left(v_{j}\right) & =\frac{\underline{T}\left(v_{j}\right)+\bar{T}\left(v_{j}\right)}{2}, \\
I\left(v_{j}\right) & =\frac{I\left(v_{j}\right)+\bar{I}\left(v_{j}\right)}{2}, \\
F\left(v_{j}\right) & =\frac{F\left(v_{j}\right)+\bar{F}\left(v_{j}\right)}{2},
\end{aligned}
$$

and 


$$
\begin{aligned}
T\left(v_{i} v_{j}\right) & =\frac{T\left(v_{i} v_{j}\right)+\bar{T}\left(v_{i} v_{j}\right)}{2}, \\
I\left(v_{i} v_{j}\right) & =\frac{I\left(v_{i} v_{j}\right)+\bar{I}\left(v_{i} v_{j}\right)}{2} \\
F\left(v_{i} v_{j}\right) & =\frac{F\left(v_{i} v_{j}\right)+\bar{F}\left(v_{i} v_{j}\right)}{2} .
\end{aligned}
$$

of each selected company and industry decision is $v_{k}$ if $v_{k}=\max _{i} S\left(v_{i}\right)$. By calculation, we have $S(T C)=0.4926, S(C C)=1.4038, S(R E)=0.0667, S(V L)=0.3833, S(A D)=0.1429$ and $S(T T)=1.3529$. Clearly, $C C$ is the optimal decision. Therefore, the carpenter company is selected to get maximum possible profit. We present our proposed method as an algorithm. This Algorithm 1 returns the optimal solution for the investment problem.

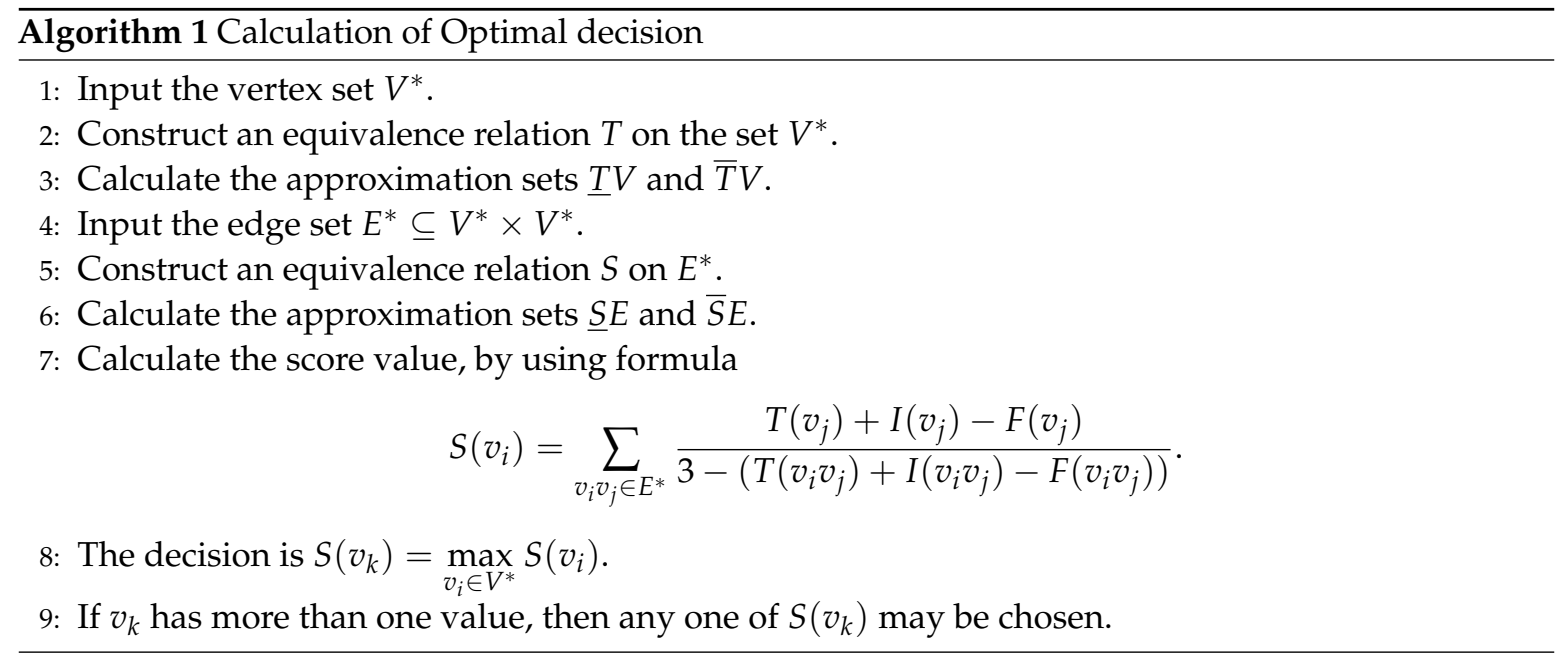

\section{Conclusions and Future Directions}

Neutrosophic sets and rough sets are very important models to handle uncertainty from two different perspectives. A rough neutrosophic model is a hybrid model which is made by combining two mathematical models, namely, rough sets and neutrosophic sets. This hybrid model deals with soft computing and vagueness by using the lower and upper approximation spaces. A rough neutrosophic set model gives more precise results for decision-making problems as compared to neutrosophic set model. In this paper, we have introduced the notion of rough neutrosophic digraphs. This research work can be extended to: (1) rough bipolar neutrosophic soft graphs; (2) bipolar neutrosophic soft rough graphs; (3) interval-valued bipolar neutrosophic rough graphs; and (4) neutrosophic soft rough graphs.

Acknowledgments: The authors are very thankful to the Editor and referees for their valuable comments and suggestions for improving the paper.

Author Contributions: Sidra Sayed and Nabeela Ishfaq conceived and designed the experiments; Muhammad Akram performed the experiments; Florentin Smarandache contributed reagents/materials/analysis tools.

Conflicts of Interest: The authors declare that they have no conflict of interest regarding the publication of the research article.

\section{References}

1. Smarandache, F. A Unifying Field in Logics. Neutrosophy: Neutrosophic Probability, Set and Logic; American Research Press: Rehoboth, NM, USA, 1999.

2. Zadeh, L.A. Fuzzy sets. Inf. Control 1965, 8, 338-353.

3. Smarandache, F. Neutrosophy Neutrosophic Probability, Set, and Logic; American Research Press: Rehoboth, NM, USA, 1998. 
4. Wang, H.; Smarandache, F.; Zhang, Y.; Sunderraman, R. Single-valued neutrosophic sets. Multispace Multistructure 2010, 4, 410-413.

5. Ye, J. Multicriteria decision-making method using the correlation coefficient under single-valued neutrosophic environment. Int. J. Gen. Syst. 2013, 42, 386-394.

6. Ye, J. Improved correlation coefficients of single valued neutrosophic sets and interval neutrosophic sets for multiple attribute decision making. J. Intell. Fuzzy Syst. 2014, 27, 2453-2462.

7. Pawlak, Z. Rough sets. Int. J. Comput. Inf. Sci. 1982, 11, 341-356.

8. Dubois, D.; Prade, H. Rough fuzzy sets and fuzzy rough sets. Int. J. Gen. Syst. 1990, 17, 191-209.

9. Liu, P.; Chen, S.M. Group decision making based on Heronian aggregation operators of intuitionistic fuzzy numbers. IEEE Trans. Cybern. 2017, 47, 2514-2530.

10. Broumi, S.; Smarandache, F.; Dhar, M. Rough neutrosophic sets. Neutrosophic Sets Syst. 2014, 3, $62-67$.

11. Yang, H.L.; Zhang, C.L.; Guo, Z.L.; Liu, Y.L.; Liao, X. A hybrid model of single valued neutrosophic sets and rough sets: Single valued neutrosophic rough set model. Soft Comput. 2016, 21, 6253-6267, doi:10.1007/s00500-016-2356-y.

12. Mordeson, J.N.; Peng, C.S. Operations on fuzzy graphs. Inf. Sci. 1994, 79, 159-170.

13. Akram, M.; Shahzadi, S. Neutrosophic soft graphs with applicatin. J. Intell. Fuzzy Syst. 2017, 32, 841-858.

14. Akram, M.; Sarwar, M. Novel multiple criteria decision making methods based on bipolar neutrosophic sets and bipolar neutrosophic graphs. Ital. J. Pure Appl. Math. 2017, 38, 368-389.

15. Akram, M.; Siddique, S. Neutrosophic competition graphs with applications. J. Intell. Fuzzy Syst. 2017, 33, 921-935.

16. Akram, M.; Sitara, M. Interval-valued neutrosophic graph structures. Punjab Univ. J. Math. 2018, 50, $113-137$.

17. Zafer, F.; Akram, M. A novel decision-making method based on rough fuzzy information. Int. J. Fuzzy Syst. 2017, doi:10.1007/s40815-017-0368-0.

18. Banerjee, M.; Pal, S.K. Roughness of a fuzzy set. Inf. Sci. 1996, 93, 235-246.

19. Liu, P.; Chen, S.M.; Junlin, L. Some intuitionistic fuzzy interaction partitioned Bonferroni mean operators and their application to multi-attribute group decision making. Inf. Sci. 2017, 411, 98-121.

20. Liu, P. Multiple attribute group decision making method based on interval-valued intuitionistic fuzzy power Heronian aggregation operators. Comput. Ind. Eng. 2017, 108, 199-212.

21. Zhang, X.; Dai, J.; Yu, Y. On the union and intersection operations of rough sets based on various approximation spaces. Inf. Sci. 2015, 292, 214-229.

22. Bao, Y.L.; Yang, H.L. On single valued neutrosophic refined rough set model and its application. J. Intell. Fuzzy Syst. 2017, 33, 1235-1248. 